\title{
Online Adaptation of Two-Parameter Inverter Model in Sensorless Motor Drives
}

This paper was downloaded from TechRxiv (https://www.techrxiv.org).

\section{LICENSE}

CC BY 4.0

SUBMISSION DATE / POSTED DATE

$21-12-2021 / 11-01-2022$

\section{CITATION}

Chen, Jiahao; Mei, Jie; Yuan, Xin; Zuo, Yuefei; Zhu, Jingwei; Lee, Christopher H. T. (2021): Online Adaptation of Two-Parameter Inverter Model in Sensorless Motor Drives. TechRxiv. Preprint.

https://doi.org/10.36227/techrxiv.17309948.v2

$\mathrm{DOI}$

10.36227/techrxiv.17309948.v2 


\title{
Online Adaptation of Two-Parameter Inverter Model in Sensorless Motor Drives
}

\author{
Jiahao Chen, Member, IEEE, Jie Mei, Member, IEEE, Xin Yuan, Member, IEEE, \\ Yuefei Zuo, Member, IEEE, Jingwei Zhu, Christopher H. T. Lee, Senior Member, IEEE
}

\begin{abstract}
This paper designs parameter adaptation algorithms for online simultaneous identification of a two-parameter sigmoid inverter model for compensating inverter nonlinearity to reduce the voltage error in flux estimation for a position sensorless motor drive. The inverter model has two parameters, $a_{2}$ and $a_{3}$, where $a_{2}$ is "plateau voltage", and $a_{3}$ is a shape parameter that mainly accounts for the stray capacitor effect. Parameter $a_{3}$ is identified by the $(6 k \pm 1)$-th order harmonics in measured current. Parameter $a_{2}$ is identified by the amplitude mismatch of the estimated active flux. It is found that the classic linear flux estimator, i.e., the hybrid of voltage model and current model, cannot be used for $a_{2}$ identification. This paper proposes to use a saturation function based nonlinear flux estimator to build an effective indicator for $a_{2}$ error. The coupled identifiability of the two parameters is revealed and analyzed, which was not seen in literature. The concept of the low current region where the two-way coupling between $a_{2}$ and $a_{3}$ occurs is established. In theory, it is suggested to stop the inverter identification in the low current region. However, the experimental results in which dc bus voltage variation and load change are imposed, have shown the effectiveness of the proposed online inverter identification and compensation method, even in low current region.
\end{abstract}

Index Terms-inverter nonlinearity model, permanent magnet motor, parameter estimation, active flux estimator.

\section{INTRODUCTION}

The voltage source inverter along with the pulse-width modulation (PWM) plays a significant role in commercialized adjustable speed motor drives. Fig. 1 shows a typical threephase three-wire inverter topology with three half-bridges. Given the fact that inverter terminal voltage sensors are absent in most motor drives, when the motor drives are operating in position sensorless mode, the commanded controller voltage $u_{x g}^{*}$ will be used instead of the actual terminal voltage $u_{x g}$ for state (e.g., active flux [1]) observation, where $g$ denotes the center of the dc bus capacitor and $x$ is the phase name placeholder, $x=a, b, c$.

In practice, non-ideal properties of the power switches, i.e., dead-time, turn-on/off time, conduction voltage drop, and stray capacitor, will result in an undesired inverter voltage drop $D_{x}\left(i_{x}\right)$ as a nonlinear function of phase current $i_{x}$, thus the inverter's actual terminal voltage $u_{x g}$ is

$$
u_{x g}=u_{x g}^{* *}-D_{x}
$$

which motivates the need of a feedforward compensation term $\hat{D}_{x}$ to cancel the inverter voltage drop $D_{x}$, and hence the commanded inverter voltage $u_{x g}^{* *}$ should be

$$
u_{x g}^{* *}=u_{x g}^{*}+\hat{D}_{x}
$$

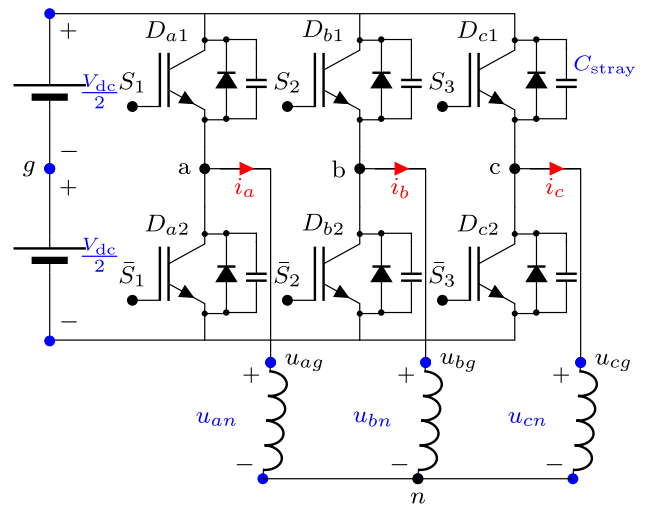

Fig. 1. Three-phase three-wire voltage-source inverter topology.

which results in the compensation error as follows

$$
u_{x g}-u_{x g}^{*}=\left(u_{x g}^{* *}-D_{x}\right)-u_{x g}^{*}=\hat{D}_{x}-D_{x} \triangleq-\tilde{D}_{x}
$$

The voltage error $\tilde{D}_{x}$ influences the performance of model based position sensorless drive in at least two aspects. First, the fundamental component in $\tilde{D}_{x}$ will cause errors in both the amplitude and angle of the estimated active flux. Second, the $(6 k \pm 1)$-th order harmonics in $\tilde{D}_{x}$ will cause fluctuation in both position and speed estimation. In addition, deteriorated performance due to $\tilde{D}_{x}$ is also observed in magnetic saliency based sensorless drives [2]-[4].

The compensation voltage $\hat{D}_{x}$ can be obtained using various methods, which can be classified into datasheet model [2], offline measurement and online compensation. The offline measurement is often executed at motor standstill, and the inverter voltage-current characteristics, i.e., the U-I curve, are recorded for look-up table (LUT) or curve fitting purposes [3], [5], [6]. Particularly in [3], both current amplitude and angle dependencies of $\hat{D}_{x}$ are measured and stored as a 2D-LUT. Besides, it is also possible to obtain the U-I curve when motor is running, e.g., at $16.67 \mathrm{~Hz}$ stator frequency, using repetitive control, which results in a U-I curve that additionally includes the hysteresis effect [7, Fig. 14].

As a matter of fact, inverter characteristics depend not only on load current but also on temperature [8]. Moreover, the dc bus voltage is not always stiff, especially for applications involving batteries. Even though the dc bus voltage can be measured, there is a nonlinear mapping between U-I curve and dc bus voltage, especially at low currents. The above facts motivate the need of online compensation.

Online compensation methods can be classified into invasive methods and non-invasive methods. Invasive methods involve 
TABLE I

Summary of DifFerent Online Parameter Adaptation Methods.

\begin{tabular}{|c|c|c|c|c|c|c|}
\hline $\begin{array}{l}\text { Adaptive } \\
\text { method }\end{array}$ & $\begin{array}{l}\text { Compensation } \\
\text { voltage model }\end{array}$ & $\begin{array}{l}\text { Unknown } \\
\text { parameter(s) }\end{array}$ & $\begin{array}{l}\text { Parameter error } \\
\text { indicator(s) }\end{array}$ & $\begin{array}{l}\begin{array}{l}\text { Known } \\
\text { parameter }\end{array} \\
\end{array}$ & $\begin{array}{l}\text { Dependent } \\
\text { variable }\end{array}$ & Compensation voltage type \\
\hline Liang et al. [14] & $\hat{D}_{x}\left(\operatorname{sign}\left(i_{x}\right) ; a_{2}\right)$ & $a_{2}$ & $d$-axis voltage command $u_{d}^{*}$ & - & $\operatorname{sign}\left(i_{x}\right)$ & Current polarity $\operatorname{sign}\left(i_{x}\right)$ based \\
\hline Inoue et al. [15] & $\hat{D}_{x}\left(i_{x} ; a_{2}, k_{S}\right)$ & $a_{2}$ & $q$-axis voltage equation & $k_{S}$ & $i_{x}$ & Current value $i_{x}$ based \\
\hline Park \& Sul [16] & $\hat{D}_{x}\left(\theta_{x} ; a_{2}, \theta_{t}\right)$ & $\theta_{t}$ & Current harmonics $I_{h}$ & $a_{2}$ & $\theta_{x}$ & Current phasor angle $\theta_{x}$ based \\
\hline Proposed & $\hat{D}_{x}\left(i_{x}^{*} ; a_{2}, a_{3}\right)$ & $a_{2}, a_{3}$ & Flux ampl. error $B$ and $I_{h}$ & - & $i_{x}$ & Current value $i_{x}^{*}$ based \\
\hline
\end{tabular}

additional excitation to the motor. In a rotating carrier signal injection-based sensorless drive, voltage error's negative effect on the position estimation can be detected from the positivesequence carrier current [4]. In [9], the dead-time is online identified by actively switching between continuous PWM and discontinuous PWM.

The non-invasive methods are further divided into invertermodel-free methods [10]-[13] and online parameter adaptation. For example, the inverter voltage drop $D_{x}$ can be reproduced by a time-delay control based disturbance observer [10] without using any inverter model. The disturbance observer method is reported to have deteriorate performance at higher speeds [11], and it relies on measured speed for calculating emf. Sensorless inverter-model-free compensation can be achieved through iterative learning [12], given the fact that the harmonics in estimated $d$-axis emf are periodic. In [13], the compensation based on single noise canceller is designed to minimize the measured 6th-order current harmonics caused by imperfect compensation. However, the inverter-model-free property means the compensation could be exact only at steady state, and there will be undesired transients in compensation voltage whenever motor operating condition changes.

In order to achieve inverter-model-based online compensation in sensorless drive, online parameter adaptation is proposed, for which an inverter model is indispensable:

- In [14], [17], [18], the square waveform compensation voltage $\hat{D}_{x}\left(\operatorname{sign}\left(i_{x}\right) ; a_{2}\right)$ depends on current polarity, and is modelled with a single plateau voltage parameter, $a_{2}$. The plateau voltage $a_{2}$ can be extracted from the commanded $d$-axis voltage that regulates the $d$-axis current to zero against the disturbance voltage caused by the inverter, if the $d$-axis position is available [17], [18]. This idea is later modified and tested in a sensorless drive [14].

- In [15], a saturation function model $\hat{D}_{x}\left(i_{x} ; a_{2}, k_{S}\right)$ is used, where the slope $k_{S}$ is assumed known, and $a_{2}$ is online identified by applying recursive least square method to the $q$-axis voltage equation.

- A trapezoidal voltage model $\hat{D}_{x}\left(\theta_{x} ; a_{2}, \theta_{t}\right)$ is proposed by Park \& Sul [16], which is mapped to the current phasor angle, $\theta_{x}$, for online compensating the inverter voltage drop. This model depends on two parameters, i.e., the ramp region angle $\theta_{t}$ and the plateau voltage $a_{2}$. By assuming $a_{2}$ is known, the $\theta_{t}$ is updated to minimize the 6th order current harmonics [16] or the sum of 6th and 12th order harmonics [19] in a synchronous $d q$-frame.

The key take-away here is that the inverter can be modeled with only two parameters, i.e., plateau voltage $a_{2}$ and shape parameter, e.g., $\theta_{t}$ or $k_{S}$. The plateau voltage parameter $a_{2}$ equals to $3 V^{\prime}$ with the distortion voltage $V^{\prime}$ defined in [20], and the shape parameter mainly accounts for effect of stray capacitor [21, Sec. 7.1.3] [22].

Table I summarizes the differences among different online parameter adaptation methods. Method in [14] relies solely on plateau voltage $a_{2}$, but the current polarity based compensation voltage is prone to suffering large voltage error when wrong polarity is detected, especially for motor with small inductance (e.g., high speed motor). In literature, only the researches in [16], [19] have identified the shape parameter, but it has two main issues that need improvement. First, there exists undesired transient process in $\theta_{t}$ whenever there is a change in current vector amplitude. This phenomenon implies that the optimal value of $\theta_{t}$ is a function of current amplitude, and is closely related to the fact that the shape of trapezoidal voltage is solely determined by $\theta_{t}$ regardless of current amplitude values. Second, the fundamental voltage error is a function of $\theta_{t}$. That is, as $\theta_{t}$ keeps increasing, there will be more and more loss in the fundamental component of the trapezoidal voltage, as shown in [16, Fig. 8].

Motivated by the two issues regarding the trapezoidal voltage method [16], this paper proposes: (i) to use current value based inverter model $\hat{D}_{x}\left(i_{x}\right)$ instead of the current phasor angle based inverter model $\hat{D}_{x}\left(\theta_{x}\right)$, and (ii) to online identify $a_{2}$ for compensating fundamental voltage error. Conventionally, $a_{2}$ is calculated from voltage equation and the current derivative must be properly dealt with. For example, [15] uses the $q$-axis voltage equation as identification model so the $q$-axis current derivative can be neglected, while the timedelay approach [10] uses the $\alpha \beta$-frame voltage equations to calculate the disturbance voltage so the current derivative needs to be approximated with numerical integration plus low-pass filter. This paper, however, integrates the voltage equation, and proposes to use a dedicated adaptive nonlinear flux estimator for online identification of $a_{2}$. Compared to [15], our proposed method does not rely on the knowledge of speed and does not assume the shape parameter is known. Compared to [10], our proposed method does not need to implement pure differentiation.

Aside from the classification based literature review above, it should be pointed out that the inverter nonlinearity compensation is really hardware-related. For example, some inverter shows very steep change in $D_{x}$ (i.e., very large $\frac{\mathrm{d} D_{x}}{\mathrm{~d} i_{x}}$ ) near $i_{x}=0$, see e.g., [23]; and in this case, extra care must be taken to obtain the correct current polarity, for example, by instantaneous back calculation [23] or by current prediction at switching instant [21, Sec. 7.1.4]. This paper, on the other hand, studies the general problem of online adaptation of 
inverter model parameters.

The contributions of this paper are, i) to analyze the parameter identifiability of an inverter model with respect to motor operating conditions; ii) to analyze the coupled identifiability for the two inverter parameters, iii) to propose a complete parameter adaptation scheme using coherent demodulation (CD) and a modified saturation based flux estimator.

\section{Proposed Online Compensation Method}

Let ${ }^{\wedge}$ and ${ }^{*}$ denote the estimated and commanded value, respectively. Assuming the current control error $i_{x}-i_{x}^{*}$ can be neglected, the proposed current value dependent compensation voltage

$$
\hat{D}_{x}\left(i_{x}^{*} ; \hat{a}_{2}, \hat{a}_{3}\right)=\hat{a}_{2} \tanh \left(\frac{\hat{a}_{3}}{2} i_{x}^{*}\right)=\hat{a}_{2}\left(\frac{2}{1+\mathrm{e}^{-\hat{a}_{3} i_{x}^{*}}}-1\right)
$$

is equipped with two online parameter adaptation algorithms (PAAs) for updating the plateau voltage estimate $\hat{a}_{2}$ and the sigmoid shape parameter estimate $\hat{a}_{3}$.

The $a_{2}$-PAA is driven by estimated flux amplitude error:

$$
\begin{aligned}
s \hat{a}_{2} & =-\gamma_{a 2} m B \\
B & \triangleq K_{\text {Active }}-\frac{1}{\tau_{\psi 2} s+1}\left\|\boldsymbol{\psi}_{2}\right\|
\end{aligned}
$$

where $s=\frac{\mathrm{d}}{\mathrm{d} t}$ is differential operator, $\boldsymbol{\psi}_{2}=\left[\psi_{\alpha 2}, \psi_{\beta 2}\right]^{\top} \in \mathbb{R}^{2}$ is the output of an active flux estimator; $B$ is defined as the dc bias in the estimated active flux modulus $\hat{K}_{\text {Active }} \triangleq\left\|\boldsymbol{\psi}_{2}\right\|$ with respect to the motor's active flux parameter $K_{\text {Active }}$; the motor operating mode variable $m$ equals to 1 for motoring and equals to -1 for regenerating; $\gamma_{a 2}$ is adaptation gain, and $\tau_{\psi 2}$ is the time constant of the low-pass filter.

The $a_{3}$-PAA is driven by current harmonics: (cf. [16], [19])

$$
\begin{aligned}
s \hat{a}_{3} & =-\gamma_{a 3}\left(w_{6} I_{6}+w_{12} I_{12}+w_{18} I_{18}\right) \\
I_{h} & =\frac{1}{\tau_{\mathrm{cd}} s+1} I_{\Sigma h} \sin \left(h \theta_{a}^{*}\right) \\
I_{\Sigma h} & \triangleq i_{\alpha} \cos \theta_{a}^{*}+i_{\beta} \sin \theta_{a}^{*} \\
\theta_{a}^{*} & =\hat{\theta}_{d}+\arctan 2\left(i_{q}^{*}, i_{d}^{*}\right)-\frac{3}{2} \pi
\end{aligned}
$$

where $I_{6}, I_{12}, I_{18}$ denote the amplitudes of the 6th, 12th and 18th order harmonic current that are obtained from the coherent demodulation in (6b) with $h=6,12,18 ; w_{6}, w_{12}, w_{18}$ are constant weights; (6c) shows that the sum of harmonic currents $I_{\Sigma h}$ is obtained by transforming $\alpha \beta$-frame current $i=\left[i_{\alpha}, i_{\beta}\right]^{\top}$ into the direct axis defined by the phase $a$ current's commanded phasor angle $\theta_{a}^{*}$ [19]; (6d) shows that $\theta_{a}^{*}$ depends on the $d q$-frame current commands $i_{d}^{*}, i_{q}^{*}$ and $\boldsymbol{\psi}_{2}$ 's angle: $\hat{\theta}_{d} \triangleq \arctan 2\left(\psi_{\beta 2}, \psi_{\alpha 2}\right) ; \gamma_{a 3}$ is adaptation gain, and $\tau_{\text {cd }}$ is the time constant of the low-pass filter.

\section{A. Two Parameter Identifiability of $a_{2}$ and $a_{3}$}

The desired system behaviors due to parameter mismatch are that the fundamental voltage error is only caused by $\hat{a}_{2}$ error, and that the $(6 k \pm 1)$-th order harmonic voltage error is solely due to $\hat{a}_{3}$ error. Unfortunately, the identifiability of the two parameter $a_{2}$ and $a_{3}$ is coupled. As a result, the adaptation gains $\gamma_{a 2}, \gamma_{a 3}$ should be carefully designed, and both PAAs

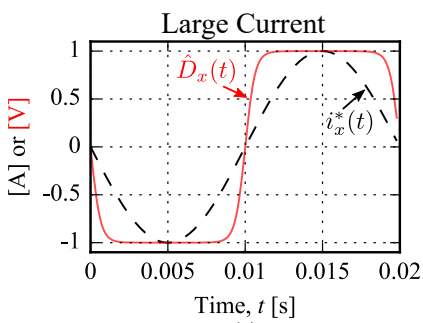

(a)

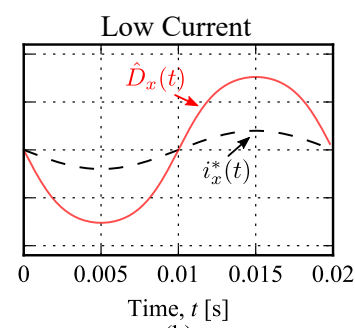

(b)
Fig. 2. The shape of compensation voltage $\hat{D}_{x}(t)=\hat{D}_{x}\left(i_{x}^{*} ; 1,10\right)$ from (4) in time domain when $i_{x}^{*}$ is a $50 \mathrm{~Hz}$ sinusoidal, i.e., $i_{x}^{*}=I_{x}^{*} \sin (50 \mathrm{~Hz} \times 2 \pi t)$. (a) $I_{x}^{*}=1 \mathrm{~A}$, (b) $I_{x}^{*}=0.2 \mathrm{~A}$. (Plots are drawn at the same scale.)

for $a_{2}$ and $a_{3}$ should be suspended when commanded phase current amplitude $I_{x}^{*}$ is too low, or more specifically when $I_{x}^{*}<6 / \hat{a}_{3}$.

1) Motivation for $a_{3}$-Identifiability: The identifiability of shape parameter $a_{3}$ depends on the harmonics in the measured $I_{\Sigma h}$. On the one hand, the identifiability of the parameter $a_{3}$ relies on the sinusoidal back emf assumption, in a sense that the $(6 k \pm 1)$-th order harmonics detected in measured current are solely due to inverter voltage error. On the other hand, the $a_{3}$-identifiability will become weak during low current region (LCR), because the compensation voltage has no $(6 k \pm 1)$-th order harmonics anymore when $I_{x}^{*}$ is too low. The time-domain shape of the phase compensation voltage $\hat{D}_{x}\left(i_{x}^{*}(t) ; \hat{a}_{2}, \hat{a}_{3}\right)$, depends on both its shape parameter $\hat{a}_{3}$ and the commanded load current $i_{x}^{*}(t)$. As shown in Fig. 2a, when $I_{x}^{*}$ is large, $\hat{D}_{x}$ in time domain is rich in harmonic contents, so that error in $\hat{a}_{3}$ causes remarkable current amplitude ripple and $a_{3}$ can be identified. On the other hand, when $I_{x}^{*}$ is very low in Fig. 2b, $\hat{D}_{x}$ in time domain looks like a pure sinusoidal, so that the error in $\hat{a}_{3}$ would cause very limited $6 k$-th order harmonics in the measured $I_{\Sigma h}$, and as a result, the PAA for $a_{3}$ should cease, because the identifiability of $a_{3}$ via current harmonics is lost.

The problem now becomes how we can decide at what current level that the PAA for $a_{3}$ should stop, and how we can generally decide this current level for any inverter. The answer to this problem is trivial (i.e., $I_{x}^{*}>0$ ) if only $a_{3}$ is being identified. However, we are going to propose that the current level for stopping $\hat{a}_{3}$ adaptation should take into account the coupled identifiability between $a_{2}$ and $a_{3}$, because when $\hat{a}_{2}$ is erroneous, $a_{3}$ cannot be identified.

2) The $a_{2}$-identifiability: The identifiability of the plateau voltage $a_{2}$ depends on the fact that using mismatched $\hat{a}_{2}$ will lead to fundamental voltage error in $\tilde{D}_{x}$ [see (3)] in state observation, and this fundamental voltage error can be revealed by calculating flux amplitude error $B$ in (5b).

3) Coupled Identifiability: Recall that $\hat{a}_{2}$ serves as a scaling factor in the two parameter inverter model (4), which implies that $\hat{a}_{2}$ error would cause both fundamental and $(6 k \pm 1)$ th order harmonic voltage error. The effect of $\hat{a}_{2}$ can be studied by plotting the harmonic contents of $\hat{D}_{x}\left(i_{x}^{*}(t) ; \hat{a}_{2}, \hat{a}_{3}\right)$ at different $\hat{a}_{2}$ values, i.e., $\hat{a}_{2}=1,2,3 \mathrm{~V}$, as shown in Fig. 3 , where the horizontal axis of $I_{x}^{*}$ is normalized by a factor of $a_{3}$. This normalization allows our analysis to be generally valid for any inverter, and implies that the effect of $\hat{a}_{3}$ and $I_{x}^{*}$ on $\hat{D}_{x}$ is equivalent.

From Fig. 3a, it is learned that the fundamental component 


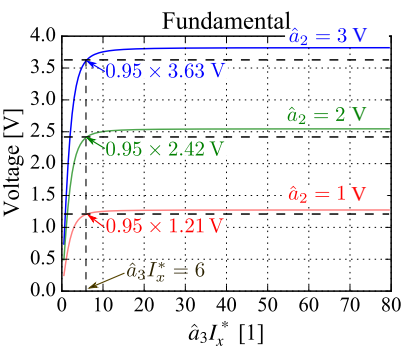

(a)

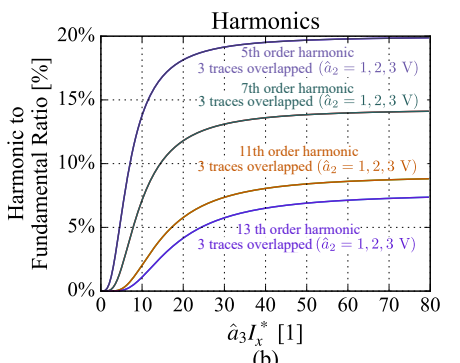

(b)
Fig. 3. Harmonic contents of the compensation voltage $\hat{D}_{x}\left(i_{x}^{*} ; \hat{a}_{2}, \hat{a}_{3}\right)$ with $i_{x}^{*}=I_{x}^{*} \sin (50 \mathrm{~Hz} \times 2 \pi t)$, plotted against the normalized current peak value $\hat{a}_{3} I_{x}^{*}$. (a) the fundamental and (b) the $(6 k \pm 1)$-th harmonic components. Note $1.21 \mathrm{~V}$ is the maximal fundamental voltage that $\hat{D}_{x}\left(\infty ; 1, \hat{a}_{3}\right)$ can provide, and the fundamental component of $\hat{D}_{x}$ being equal to $95 \%$ of its maximum always corresponds to $\hat{a}_{3} I_{x}^{*}=6$.

voltage is simply scaled by $\hat{a}_{2}$. From Fig. $3 \mathrm{~b}$, it is seen that the ratio of harmonic to fundamental does not depend on $\hat{a}_{2}$ at all, as the 3 traces at different $\hat{a}_{2}$ values of $1,2,3 \mathrm{~V}$ overlap for each harmonic. In summary, $\hat{a}_{2}$ acts as scaling factor, and $\hat{a}_{2}$ error would result in voltage error $\tilde{D}_{x}$ at all harmonics; while a change in $\hat{a}_{3}$ will mainly cause a change in harmonics contents in $\hat{D}_{x}$ if $\hat{a}_{3} I_{x}^{*}$ is large enough.

As a result, both $\hat{a}_{2}$ error and $\hat{a}_{3}$ error could lead to harmonics in the measured $I_{\Sigma h}$, which means that the two parameter identifiability is coupled in terms of current harmonics. When the proposed PAAs are implemented in practice, erroneous $\hat{a}_{2}$ would cause diverged $\hat{a}_{3}$ results.

To enable decoupled identification, the convergence of $\hat{a}_{2}$ is of higher priority, and $\gamma_{a 2}$ should be selected to be relatively large. After $\hat{a}_{2}$ fast converges, the convergence of $\hat{a}_{3}$ is then guaranteed by its single-parameter-identifiability.

4) Loss of $a_{2}$-Identifiability in Low $\hat{a}_{3} I_{x}^{*}$ Region: Unfortunately, the decoupled identification is not effective for all working conditions. According to Fig. $3 \mathrm{a}$, for a fixed $\hat{a}_{2}$ value, the fundamental component in $\hat{D}_{x}(t)$ drastically decreases as $\hat{a}_{3} I_{x}^{*}$ reduces below 6 , and equals to $95 \% \times 1.21 \hat{a}_{2}$ when $\hat{a}_{3} I_{x}^{*}=6$. It is desired that the fundamental component in $\hat{D}_{x}(t)$ always equals to $1.21 \hat{a}_{2}$ for any $\hat{a}_{3} I_{x}^{*}$. However, when $\hat{a}_{3} I_{x}^{*}<6$ (i.e., when current is low), erroneous $\hat{a}_{3}$ will lead to biased $\hat{a}_{2}$ results. This is because during low $\hat{a}_{3} I_{x}^{*}$ region, erroneous $\hat{a}_{3}$ is also causing fundamental voltage error in $\tilde{D}_{x}$.

As a result, during low $\hat{a}_{3} I_{x}^{*}$ region, both $\hat{a}_{2}$ error and $\hat{a}_{3}$ error could lead to fundamental error in $\tilde{D}_{x}$ for state observation, which means the two parameter identifiability becomes coupled in terms of the flux amplitude error (or fundamental voltage error). Since we cannot differentiate the fundamental voltage error contribution between $\hat{a}_{2}$ and $\hat{a}_{3}$, the PAAs should stop when $\hat{a}_{3} I_{x}^{*}<6$.

\section{B. Main Proposition}

Taking the two parameter identifiability into account, this paper proposes to stop both PAAs when the normalized commanded current peak value $\hat{a}_{3} I_{x}^{*}$ is less than 6 , which corresponds to the situation where the fundamental component of $\hat{D}_{x}(t)$ has reduced down to $95 \%$ due to reduction in $\hat{a}_{3} I_{x}^{*}$.

Main Proposition: The PAAs (5a) and (6a) should be implemented with their adaptation gains satisfying the following

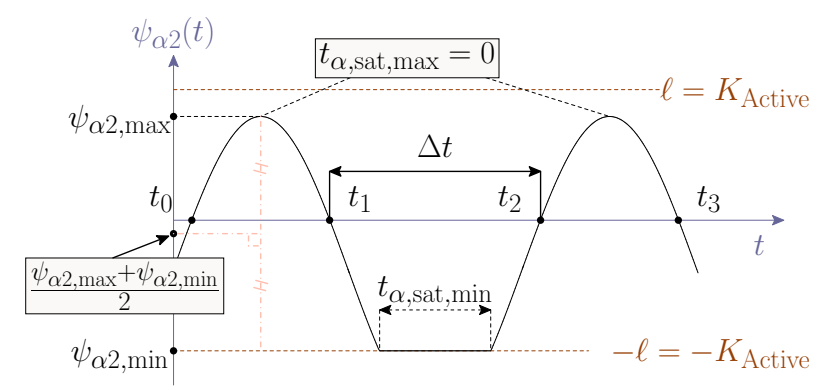

Fig. 4. Graphical definitions of symbols after Sat(.) is introduced for $\boldsymbol{\psi}_{2}$.

requirements:

(Fast $\hat{a}_{2}$ convergence) $\gamma_{a 2} \gg \gamma_{a 3}$, if $\gamma_{a 2}, \gamma_{a 3} \neq 0$,

(Loss of $a_{2}$-identifiability) $\gamma_{a 2}=0$, if $\hat{a}_{3} I_{x}^{*}<6$,

(Loss of $a_{3}$-identifiability) $\gamma_{a 3}=0$, if $\hat{a}_{3} I_{x}^{*}<6$.

In summary, the two parameter identifiability is revealed in terms of the single parameter identifiability and the decoupled identifiability for each parameter as follows. The $a_{2}$ parameter identifiability is understood by

- the fact that $\hat{a}_{2}$ error will cause fundamental error in $\tilde{D}_{x}$ that leads to flux amplitude error $B$;

- and the assumption that current $\hat{a}_{3} I_{x}^{*}$ is large enough such that there is less than 5\% reduction in fundamental component of $\hat{D}_{x}(t)$ at any fixed $\hat{a}_{2}$ value, as is indicated in Fig. 3a. This imposes (7b).

The $a_{3}$ parameter identifiability is understood by

- the fact that when current $\hat{a}_{3} I_{x}^{*}$ is large enough, $\hat{a}_{3}$ error will cause harmonics error in $\tilde{D}_{x}$ that will lead to remarkable harmonics in the measured $I_{\Sigma h}(6 \mathrm{c})$; but when current $\hat{a}_{3} I_{x}^{*}$ becomes lower and lower, the harmonics contents gradually vanish, as shown in Fig. 3b;

- and the assumption that $\hat{a}_{2}$ has almost converged such that the harmonics detected in the measured $I$ are mainly due to $\hat{a}_{3}$ error rather than $\hat{a}_{2}$ error. This imposes (7a).

Note from Fig. 3b, (7c) can be relaxed to lower value than 6 if only $a_{3}$ is being identified. However, since $a_{3}$-identifiability assumes perfect knowledge of $a_{2}, a_{3}$-PAA should be also suspended when $a_{2}$-PAA stops to update.

\section{The Nonlinear Active Flux Estimator}

The PAA (5a) for $a_{2}$ relies on a flux estimator that can convert voltage error $\tilde{D}_{x}$ into nonzero dc bias $B$ in $\left\|\psi_{2}\right\|$. According to our study, the classical linear flux estimator (e.g., the voltage model and current model fusion method [24]) fails to produce nonzero dc bias $B$, (which implies that $a_{2}$ cannot be identified from $B$,) when the drive is regenerating $(m=-1)$ and $\hat{a}_{2}<a_{2}$ in Fig. 5a, or when the drive is motoring $(m=1)$ and $\hat{a}_{2}>a_{2}$ in Fig. 5b. This undesired $B=0$ phenomenon is due to the proportional-integral (PI) correction terms used in the flux estimator. The PI correction is always active and is forcing the amplitude mismatch between voltage model and current model to be zero, which is to blame for the $B=0$ phenomenon.

As an alternative, we proposes to use a nonlinear flux estimator whose saturation correction action is not always triggered. In particular, the saturation function based flux estimator that originates in [25] is modified for producing 


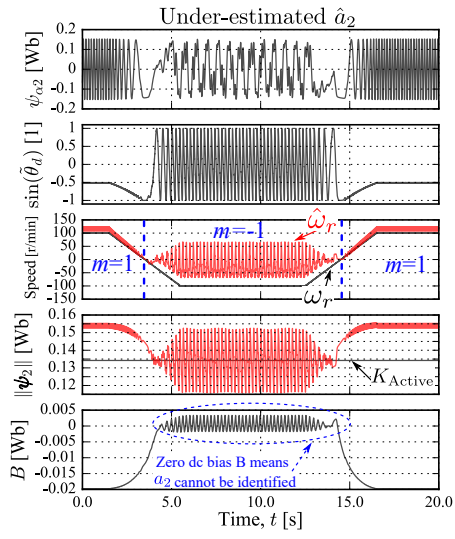

(a)

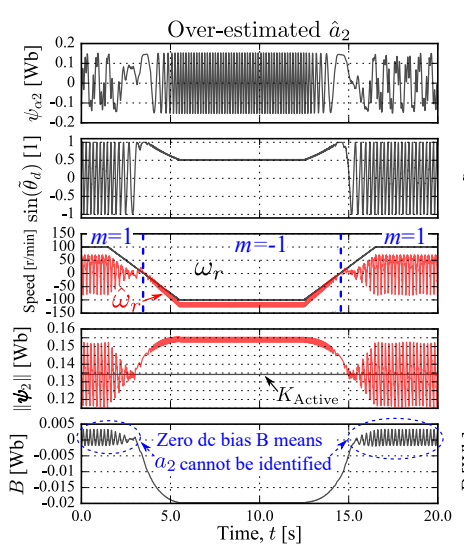

(b)

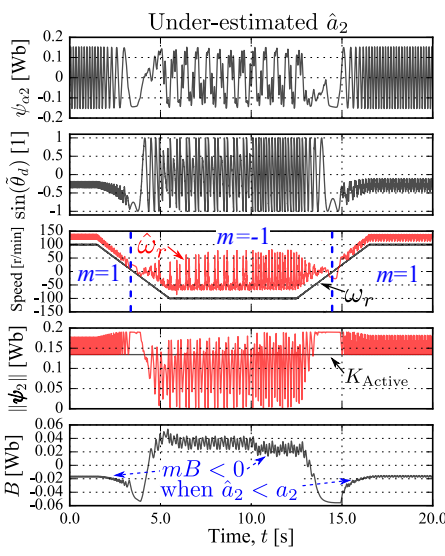

(a)

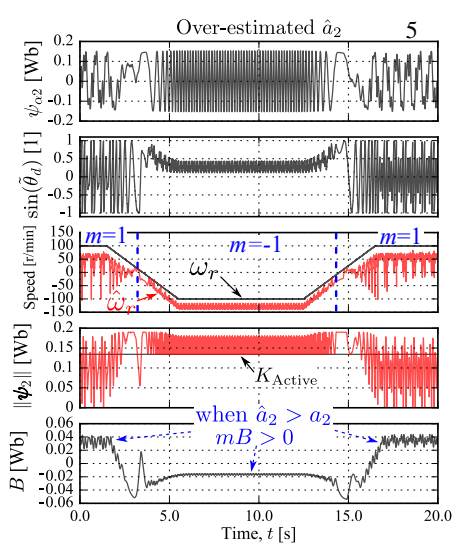

(b)

Fig. 5. Simulated speed-sensored control against positive load with $\hat{a}_{2}$ error. Fig. 6 . Simulated speed-sensored control against positive load with $\hat{a}_{2}$ error The classic Linear flux estimation [24] is implemented but not used for control. The proposed Nonlinear flux estimation (8) is implemented but not used for (a) $\hat{a}_{2}=0.8 a_{2}$, (b) $\hat{a}_{2}=1.2 a_{2}$. Note $a_{2}$ cannot be identified, if the average control. (a) $\hat{a}_{2}=0.8 a_{2}$, (b) $\hat{a}_{2}=1.2 a_{2}$. Note $a_{2}$ can be identified if $m B$ value of $\left\|\boldsymbol{\psi}_{2}\right\|$ is equal to $K_{\text {Active }}$, i.e., $B=0$.

a non-zero $B$. The key property is that the nonlinear flux estimator behaves as a pure integrator as long as the flux estimate conponent $\psi_{\alpha 2}\left(\right.$ or $\left.\psi_{\beta 2}\right)$ is within the range $[-\ell, \ell]$.

\section{A. Saturation Function based Flux Estimator}

A saturation function Sat $(\cdot)$ is added to the voltage model active flux estimator that is corrected by an offset voltage estimate $\hat{\boldsymbol{u}}_{\text {offset }}=\left[\hat{u}_{\alpha, \text { offset }}, \hat{u}_{\beta, \text { offset }}\right]^{\top}$ :

$$
\boldsymbol{\psi}_{2}=\operatorname{Sat}\left(\frac{1}{s}\left(\boldsymbol{u}^{*}-R \boldsymbol{i}-\hat{\boldsymbol{u}}_{\mathrm{offset}}\right)-L_{q} \boldsymbol{i}\right)
$$

where $\boldsymbol{u}^{*}=\left[u_{\alpha}^{*}, u_{\beta}^{*}\right]^{\top}$ is $\alpha \beta$-frame voltage command, $\boldsymbol{i}=$ $\left[i_{\alpha}, i_{\beta}\right]^{\top}$ is measured $\alpha \beta$-frame current, $R$ is stator resistance, $L_{q}$ is $q$-axis inductance, and $\operatorname{Sat}\left(\left[x_{\alpha}, x_{\beta}\right]^{\top}\right)$ limits its input vector's components $x_{\alpha}, x_{\beta}$ within the range $[-\ell, \ell]$, i.e.,

$$
\operatorname{Sat}\left(\left[\begin{array}{l}
x_{\alpha} \\
x_{\beta}
\end{array}\right]\right)=\left[\begin{array}{l}
\min \left(\max \left(x_{\alpha},-\ell\right), \ell\right) \\
\min \left(\max \left(x_{\beta},-\ell\right), \ell\right)
\end{array}\right]
$$

Ideally, the limit $\ell$ is set to $K_{\text {Active }}$. To understand the working principles of (8), consider an example scenario, where the $\alpha$ axis offset voltage error $\tilde{u}_{\alpha, \text { offset }}=u_{\alpha, \text { offset }}-\hat{u}_{\alpha, \text { offset }}$ exists and is negative. After integration, the negative $\tilde{u}_{\alpha, \text { offset }}$ will lower the entire waveform of $\psi_{\alpha 2}$, such that the lower bound $(-\ell)$ is reached, as shown in Fig. 4. From Fig. 4, $t_{0}, t_{2}$ are used to denote the time instants of the flux estimate zero-crossings, and the $\alpha$-axis flux estimate extrema are defined by

$$
\left\{\begin{array}{l}
\psi_{\alpha 2, \min }=\min _{t \in\left[t_{0}, t_{2}\right]} \psi_{\alpha 2}(t) \\
\psi_{\alpha 2, \max }=\max _{t \in\left[t_{0}, t_{2}\right]} \psi_{\alpha 2}(t)
\end{array}\right.
$$

which will be used to build the offset voltage estimate $\hat{\boldsymbol{u}}_{\text {offset }}$ to close a feedback loop that eliminates the unknown dc bias $\boldsymbol{u}_{\text {offset }}$ in the calculated emf.

\section{B. Proposed Exact Offset Voltage Calculation Method}

This paper proposes to exploit the "saturation time" to directly compute the value of offset voltage error. Graphical definitions of the saturation time concept are presented in Fig. 4 , where $t_{\alpha \text {,sat,min }}$ denotes the time duration when $\psi_{\alpha 2}$ reaches the lower bound $(-\ell)$ within $\left[t_{0}, t_{2}\right]$. Since in Fig. 4 , only the lower bound $(-\ell)$ is reached, so the lower bound saturation time $t_{\alpha, \text { sat,min }} \neq 0$ and the upper bound saturation

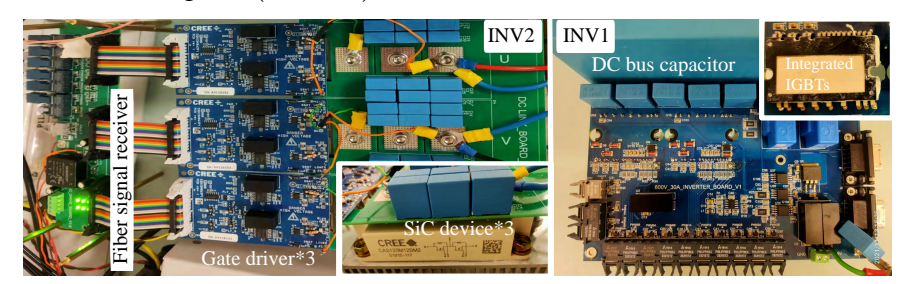

Fig. 7. Photo of the two tested inverter. Power switches are at the bottom of the printed circuit board for effective heat dissipation.

time $t_{\alpha, \text { sat,max }}=0$. With $\alpha$-axis saturation times available, the $\alpha$-axis offset voltage error can be directly calculated as

$$
\tilde{u}_{\alpha, \text { offset }}=\frac{\frac{1}{2}\left(\psi_{\alpha 2, \text { min }}+\psi_{\alpha 2, \max }\right)}{t_{2}-t_{0}-t_{\alpha, \text { sat }, \text { min }}-t_{\alpha, \text { sat, } \max }}
$$

which means that "it takes $\left(t_{2}-t_{0}-t_{\alpha, \text { sat,min }}-t_{\alpha, \text { sat,max }}\right)$ [s] for the uncompensated offset voltage $\tilde{u}_{\alpha, \text { offset }}[\mathrm{V}]$ to contribute to a flux dc bias of $\frac{1}{2}\left(\psi_{\alpha 2, \min }+\psi_{\alpha 2 \text {,max }}\right)[\mathrm{Wb}]$ in the $\alpha$-axis flux estimate $\psi_{\alpha 2}$."

Finally, our proposed offset voltage estimate for $\alpha$-axis is

$$
\hat{u}_{\alpha, \text { offset }}=\frac{k_{i}}{s} \tilde{u}_{\alpha, \text { offset }}=\frac{k_{i}}{s} \frac{\frac{1}{2}\left(\psi_{\alpha 2, \min }+\psi_{\alpha 2, \max }\right)}{t_{2}-t_{0}-t_{\alpha, \mathrm{sat}, \min }-t_{\alpha, \mathrm{sat}, \max }}
$$

with $k_{i}$ a positive scalar. The analysis for $\beta$-axis is the same as $\alpha$-axis, and $\hat{u}_{\beta, \text { offset }}$ is defined accordingly. This means the nonlinear flux estimator only introduces component-wise correction, while the flux amplitude based correction used in the conventional linear flux estimator depends on both $\alpha$-axis and $\beta$-axis components of the flux estimate.

\section{Simulated Waveforms of DC Bias B}

The simulation results of $\boldsymbol{\psi}_{2}$ from (8) using $\hat{\boldsymbol{u}}_{\text {offset }}$ from (12) are shown in Fig. 6. It is observed that "the $\hat{a}_{2}$ error indicator", $m B$, is negative when $\tilde{a}_{2}=a_{2}-\hat{a}_{2}>0$ in Fig. 6a, while $m B$ is positive when $\tilde{a}_{2}<0$ in Fig. $6 \mathrm{~b}$. This reveals the working principle of the PAA (5a), i.e., to update $\hat{a}_{2}$ by driving the error indicator $m B$ to zero.

\section{InVerter Characteristics Measurement}

Two self-built inverters shown in Fig. 7 are tested in this section and will be referred to as "INV1" and "INV2" in the sequel. 


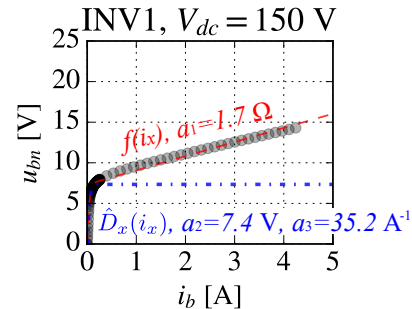

(a)

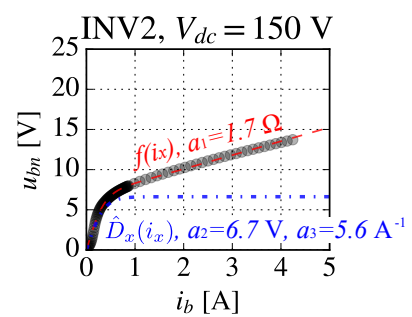

(c)

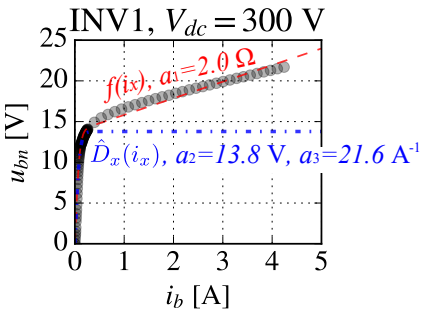

(b)

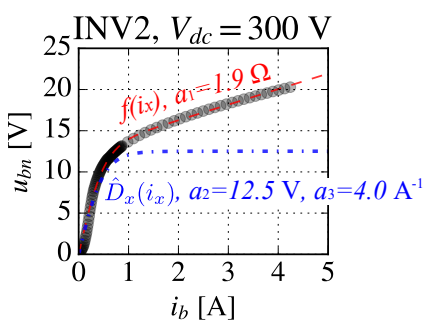

(d)
Fig. 8. U-I curves, i.e., voltage-current characteristics, of the two test inverters, (ab) INV1 and (cd) INV2, at dc bus voltages of (ac) $150 \mathrm{~V}$ and (bd) $300 \mathrm{~V}$.

- INV1 uses the $600 \mathrm{~V}, 30 \mathrm{~A}$, insulated-gate bipolar transistor (IGBT) based intelligent power module, FNB43060T2 from ON Semiconductor.

- INV2 uses the $1200 \mathrm{~V}, 120 \mathrm{~A}$, silicon carbide (SiC) metal-oxide-semiconductor field-effect transistor (MOSFET), CAS120M12BM2 from Cree.

\section{A. Offline Measurement and Curve Fitting Results}

The U-I curves of INV1 and INV2 are measured at $V_{\mathrm{dc}}=$ $150 \mathrm{~V}, 300 \mathrm{~V}$, as shown in Fig. 8. The measured data are fitted to function $f\left(i_{x} ; a_{1}, a_{2}, a_{3}\right)=a_{1} i_{x}+\hat{D}_{x}\left(i_{x} ; a_{2}, a_{3}\right)$. If the fitting error is small enough, we can use $\hat{D}_{x}$ from (4) with the fitted $a_{2}$ and $a_{3}$ as the inverter model at a certain dc bus voltage in replace of an LUT, for both INV1 and INV2. However, the fitted $a_{1}$ values annotated in Fig. 8, are found to be much larger than motor nominal resistance $(R=1.1 \Omega)$, and compensation using the fitted $a_{2}$ and $a_{3}$ leads to deteriorated sensorless control performance.

\section{B. Low Current Region (LCR) and Current Rating Matching}

From Fig. 8, it is found that $a_{2}$ increases and $a_{3}$ decreases as the dc bus voltage $V_{\mathrm{dc}}$ gets higher. Besides, INV2, whose current rating is four times as large as that of INV1, has much smaller $a_{3}$ value, and has much wider low current region (LCR), up to approximately $1.5 \mathrm{~A}$ at $V_{\mathrm{dc}}=300 \mathrm{~V}$. Here, LCR means the $i_{x}$ range (denoted by $\left[0, I_{\mathrm{LCR}}\right]$ ) in which $\hat{D}_{x}\left(i_{x}\right)$ is not a horizontal line.

According to the parametric Fourier analysis in Fig. 3, the upper bound of LCR can be estimated by the equation: $a_{3} I_{\mathrm{LCR}}=6$. When $V_{\mathrm{dc}}=300 \mathrm{~V}$, the $I_{\mathrm{LCR}}$ values of INV1 and INV2, based on the curve fitting results, are $0.3 \mathrm{~A}$ and $1.5 \mathrm{~A}$, respectively, which can be visually justified with Fig. 8. The upper bounds of the LCRs of INV1 and INV2 are approximately $1.0 \%$ and $1.25 \%$ of the rated current of the power switches, respectively. The above fact implies that the proposed PAAs should be generally applicable if the inverter current rating and the motor current rating are well matched, in which case, the motor's phase peak current $I_{x}^{*}$ will be larger

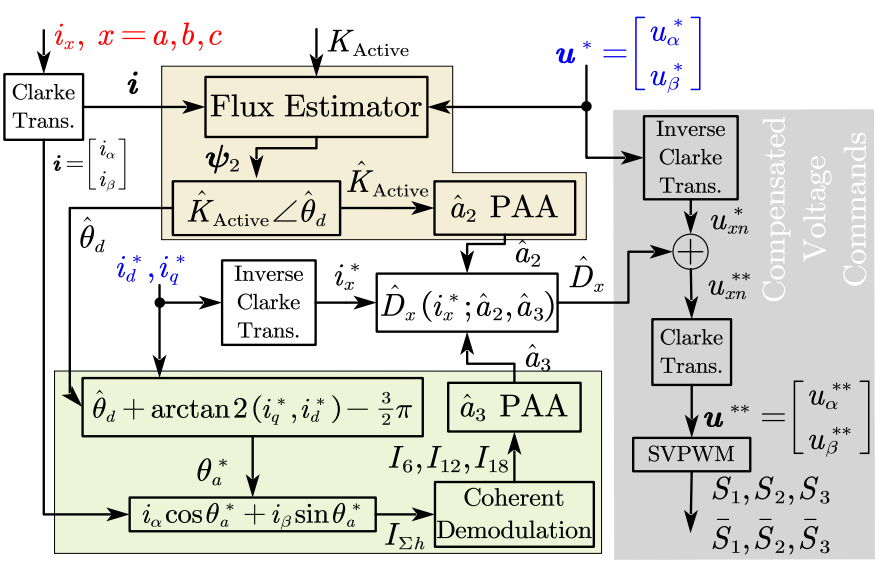

Fig. 9. Block diagram of the proposed online inverter parameter adaptation based inverter nonlinearity compensation method.

than $I_{\mathrm{LCR}}$ only to fight against friction torque. For this ideal situation, the mechanism to avoid parameter adaptation during loss of two parameter identifiability in our main proposition will not be needed.

\section{Experimental VALidation of the Proposed PAAs}

This paper investigates the challenging situation where the motor's peak current $I_{x}$ is near $I_{\mathrm{LCR}}$, such that normalized peak current $a_{3} I_{x}$ is near 6 . This is achieved by using a test motor whose rated current is much lower than the inverter current rating. By doing this, we are going to show that (7b) and $(7 \mathrm{c})$ in our main proposition are conservative, and the PAAs are found to be effective down to much lower current than $\hat{a}_{3} I_{x}^{*}=6$.

\section{A. Test Bench Setup}

INV1 and INV2 drive two $750 \mathrm{~W}, 3$ Arms, 4 pole pairs, 3phase surface-mounted permanent magnet servo motors whose shafts are mechanically coupled. A dc power supply is used as the shared dc bus for both inverters. The period of the space vector pulse-width modulation (SVPWM) is $T_{\mathrm{PWM}}=0.1 \mathrm{~ms}$. Dead-time is $5 \mu$ s in Fig. 10 and Fig. 11.

INV2 drives the test motor, and INV1 drives the load motor. The parameters of the two motors are: $R=1.1 \Omega, d$-axis inductance $L_{d}=5 \mathrm{mH}=L_{q}$, permanent magnet flux linkage $K_{E}=0.1 \mathrm{~Wb}$. By definition we have $K_{\text {Active }}=K_{E}+$ $\left(L_{d}-L_{q}\right) i_{d}$ [1]. The load motor is vector controlled with a fixed speed command of $-300 \mathrm{r} / \mathrm{min}$ and its speed regulator output limit $i_{q, \max }^{*}$ is set to $3 \mathrm{~A}$ in Fig. 10 and is switched between $3 \mathrm{~A}$ and $1.5 \mathrm{~A}$ every $8 \mathrm{~s}$ in Fig. 11.

\section{B. Block Diagram and System Synthesis}

The block diagram of the PAAs based inverter voltage drop compensation scheme is shown in Fig. 9. The SVPWM module outputs gate signals to control the inverter based on the input voltage command $\boldsymbol{u}^{* *}$ as well as the measured $V_{\mathrm{dc}}$. Amplitude-invariant Clarke transformation converts phase quantities $u_{x n}^{* *}, x=a, b, c$, into $\boldsymbol{u}^{* *}$, where $u_{x n}^{* *}$ consists of the torque controller's output voltage $u_{x n}^{*}$ and the compensation voltage $\hat{D}_{x}$. Note $\hat{D}_{x}$ is a function of phase current command $i_{x}^{*}$ and is parameterized by the two inverter parameters, $\hat{a}_{2}$ and 

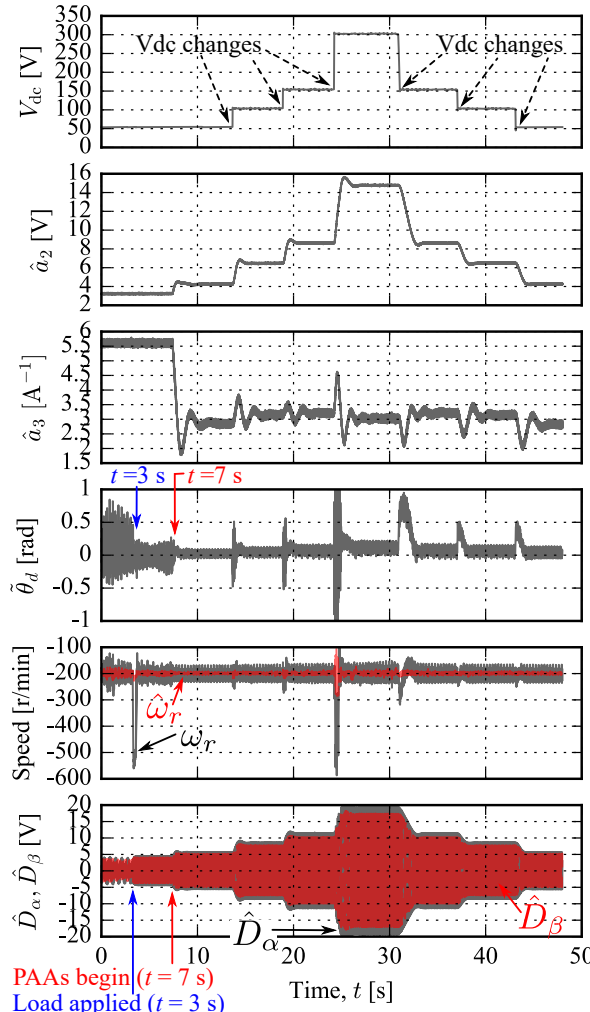

(a)
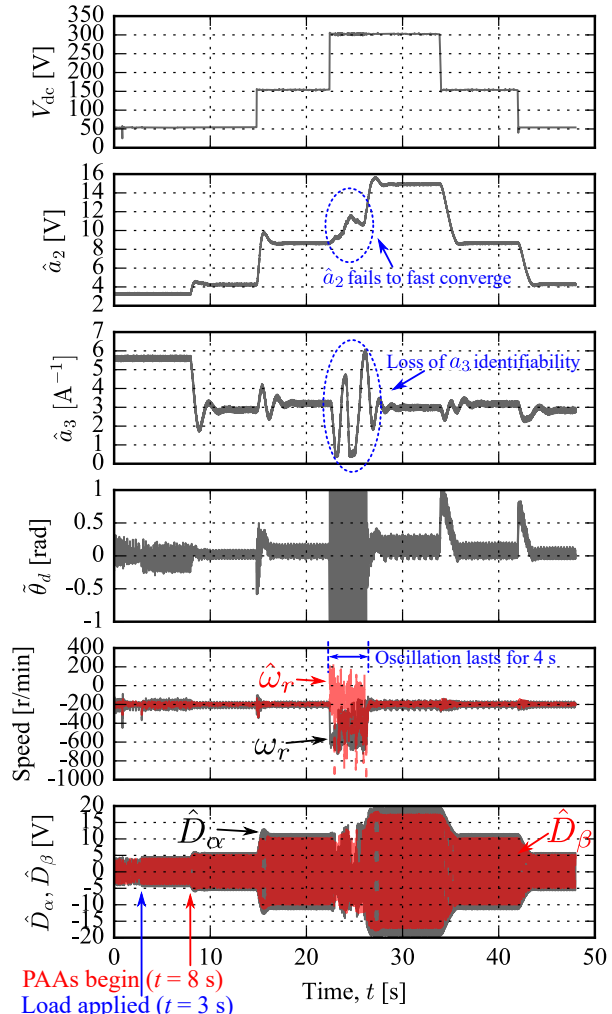

(b)
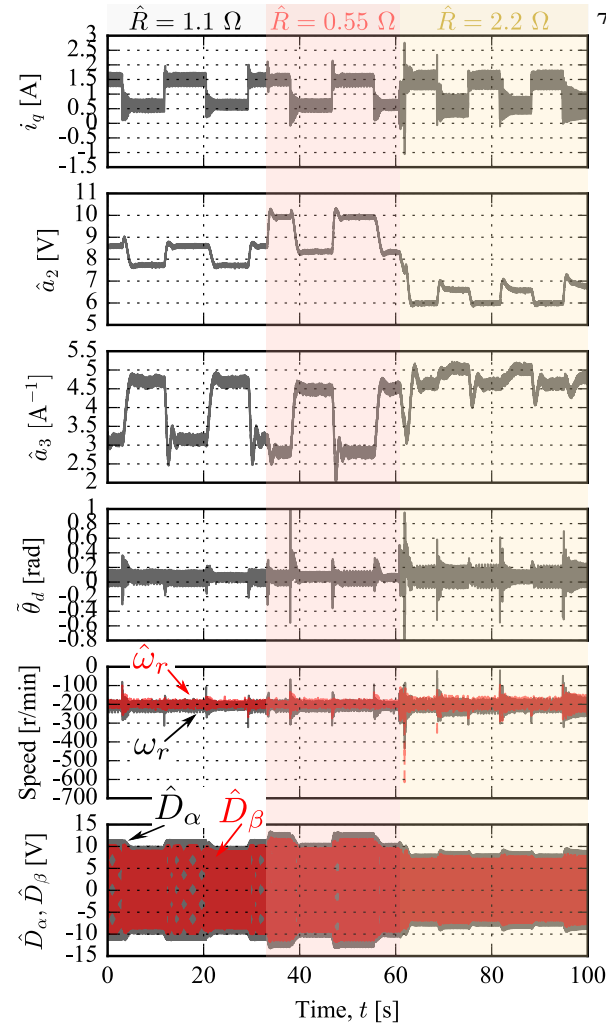

Fig. 11. Experimental sensorless operation with using the proposed PAAs at different loads with $\omega_{\mathrm{ob}}=150 \mathrm{rad} / \mathrm{s}$. The load motor's torque current switches between $1.5 \mathrm{~A}$ and $3 \mathrm{~A}$ every $8 \mathrm{~s}$.

$\hat{a}_{3}$, where $\hat{a}_{2}$ is updated by PAA in (5a) and $\hat{a}_{3}$ by PAA in (6a).

The PAA for $\hat{a}_{2}$ relies on $\hat{K}_{\text {Active }}$, the amplitude of the active flux estimate $\psi_{2}$ in (8), and the pre-identified parameter $K_{\text {Active }}$. The PAA for $\hat{a}_{3}$ depends on the detection of the 6th, 12th and 18th order current harmonics in $I_{\Sigma h}$, the direct-axis component of measured current $i$ in the commanded current vector-oriented frame from (6b). The total computational cost for executing the two PAAs in a digital signal processor with system clock frequency of $200 \mathrm{MHz}$ is $4.3 \mu \mathrm{s}$, and becomes $3.1 \mu \mathrm{s}$ if $w_{12}=w_{18}=0$.

Finally, a critically-damped cascaded speed observer (see, e.g., [26]) tuned by its observer bandwidth parameter $\omega_{\text {ob }}$, is implemented to reconstruct electrical speed $\hat{\omega}_{r}$ from $\hat{\theta}_{d}$.

\section{Adaptation of Two Parameters at Various $V_{\mathrm{dc}}$ Values}

The experimental results of sensorless operation with PAAs using INV1 at various dc bus voltage values, are shown in Fig. 10, where experimentally recorded waveforms at different speed observer bandwidths $\left(\omega_{\mathrm{ob}}\right)$ are compared.

1) Low Speed Observer Bandwidth: Fig. 10a corresponds to the case of the low speed observer bandwidth $\omega_{\mathrm{ob}}=$ $30 \mathrm{rad} / \mathrm{s}$. At beginning $(t=0)$ when $V_{\mathrm{dc}}=50 \mathrm{~V}$, position error $\tilde{\theta}_{d}=\theta_{d}-\hat{\theta}_{d}$ is oscillating and is peaking over 0.5 rad, because initial values of $\hat{a}_{2}$ and $\hat{a}_{3}$ are erroneous. The oscillation in $\tilde{\theta}_{d}$ is reduced when the motor is loaded at $t=3 \mathrm{~s}$, and is minimized at $t=7 \mathrm{~s}$ when the PAAs are applied to update $\hat{a}_{2}$ and $\hat{a}_{3}$. At $t=14,19,25 \mathrm{~s}, V_{\mathrm{dc}}$ is increased to $100,150,300 \mathrm{~V}$, respectively. It can be seen that large $V_{\mathrm{dc}}$ change (from $150 \mathrm{~V}$ to $300 \mathrm{~V}$ ) causes a large oscillation in both $\tilde{\theta}_{d}$ and the encoder measured speed signal $\omega_{r}$. After $t=30 \mathrm{~s}$,
$V_{\mathrm{dc}}$ decreases to $150,100,50 \mathrm{~V}$ in order. The waveform of $\hat{a}_{2}$ shows analogy to that of $V_{\mathrm{dc}}$.

When $\omega_{\mathrm{ob}}=30 \mathrm{rad} / \mathrm{s}$ is relatively low, the speed controller's load disturbance rejection ability is poor, but the sensorless system shows robustness against sudden voltage error $\tilde{D}_{x}$ caused by step change in $V_{\mathrm{dc}}$, such that the oscillation in speed waveform quickly diminishes in Fig. 10a.

2) High Speed Observer Bandwidth: Fig. 10b further shows the results when $\omega_{\mathrm{ob}}=150 \mathrm{rad} / \mathrm{s}$. When $\omega_{\mathrm{ob}}$ is large, the load rejection ability is improved but the robustness against voltage error disturbance is degraded. On the one hand, the spike in actual speed waveform is almost eliminated when load is applied at $t=3 \mathrm{~s}$. On the other hand, the system falls into severe oscillation for approximately $4 \mathrm{~s}$, when $V_{\mathrm{dc}}$ steps from $150 \mathrm{~V}$ to $300 \mathrm{~V}$. The oscillation is caused by the slow convergence of $\hat{a}_{2}$ as well as the oscillation in $\hat{a}_{3}$ waveform, because the identifiability of $a_{2}$ and $a_{3}$ is coupled in terms of current harmonics if $\hat{a}_{2}$ does not quickly converges, as is analyzed in Sec.II-A3.

\section{Adaptation of Two Parameters Against Load Changes}

The experiment in Fig. 10 is continued with the dc bus voltage fixed at $150 \mathrm{~V}$, but the load motor's torque current now switches between $1.5 \mathrm{~A}$ and $3 \mathrm{~A}$ every $8 \mathrm{~s}$, to test the PAAs' behavior under load change:

- When load motor torque current is $1.5 \mathrm{~A}$, the test motor current is about $I_{x} \approx 0.5 \mathrm{~A}$,

- When load motor torque current is $3 \mathrm{~A}$, the test motor current is about $I_{x} \approx 1.5 \mathrm{~A}$,

as shown in the first waveform in Fig. 11. Recall the LCR upper bound of the test inverter, INV1, is $I_{\mathrm{LCR}}=1.5 \mathrm{~A}$. 


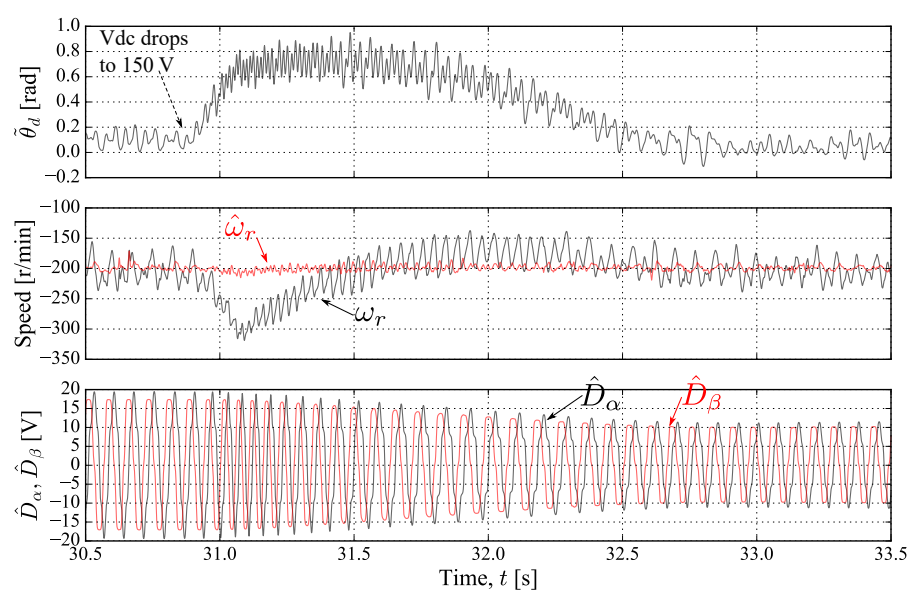

(a)
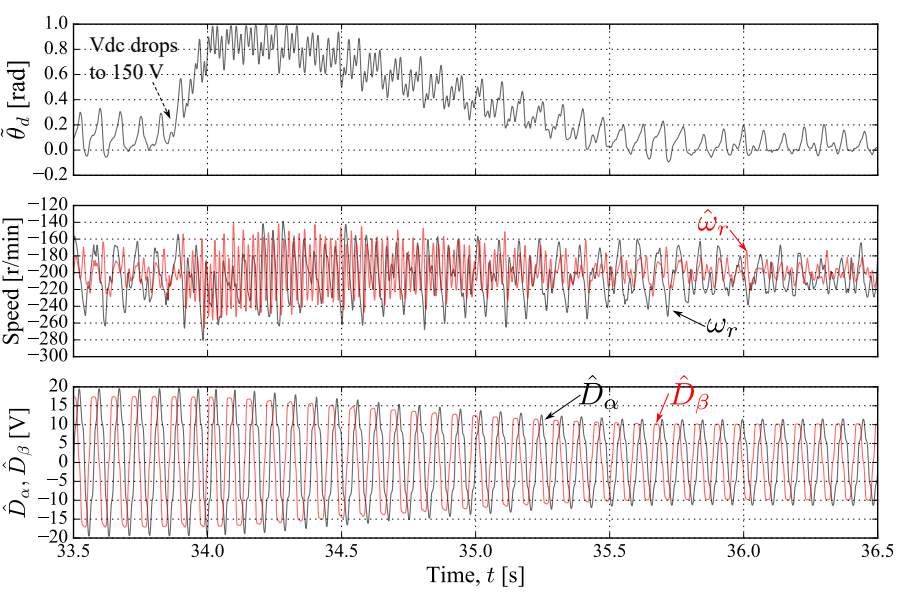

(b)

Fig. 12. Zoomed-in plot of: (a) Fig. 10a, and (b) Fig. 10b showing the transient details when dc bus voltage drops.
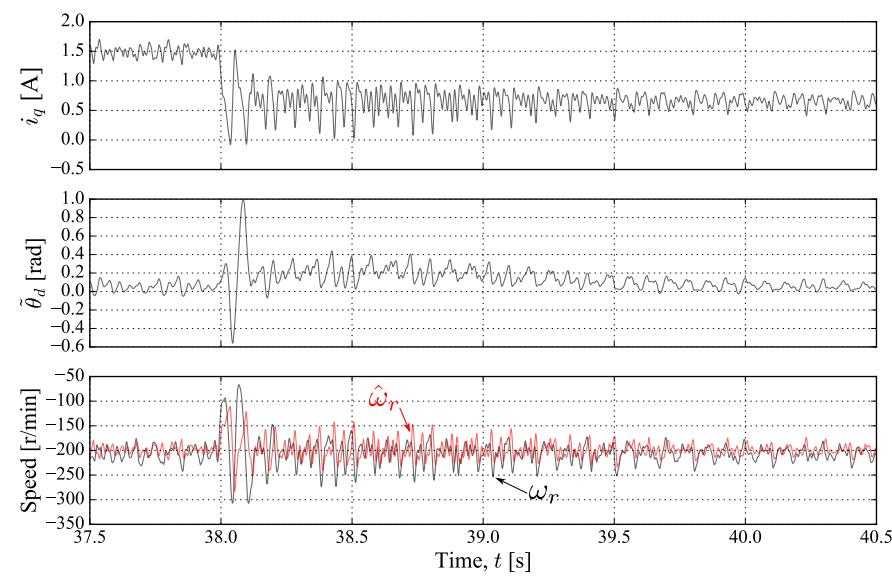

Fig. 13. Zoomed-in plot of Fig. 11 when motor torque current changes.

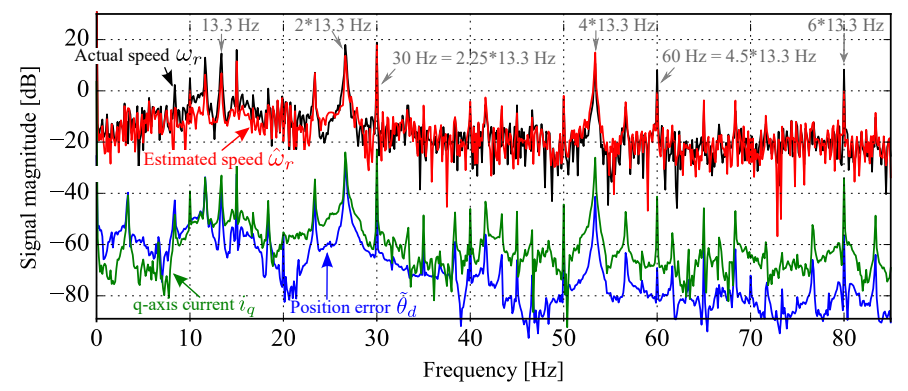

Fig. 14. Fourier analysis of the steady state waveforms in Fig. 11 when $i_{q}=1.5 \mathrm{~A}$. Note the synchronous frequency is $13.3 \mathrm{~Hz}$.

In other words, the experiment in Fig. 11 further investigates the effectiveness of the proposed PAAs under the non-ideal situation where peak current $I_{x} \approx 0.5 \mathrm{~A}$, which is less than $I_{\mathrm{LCR}}=1.5 \mathrm{~A}$.

When $t \in[0,33] \mathrm{s}$, the nominal value of resistance $R=1.1 \Omega$ is used in (8). It is observed that $\hat{a}_{2}$ converges to different values corresponding to different loads. This is an expected behavior, because $\hat{a}_{2}$ and $\hat{a}_{3}$ are coupled when motor peak current $I_{x}$ is lower than $I_{\mathrm{LCR}}$. By "coupled", we mean that both $\hat{a}_{2}$ and $\hat{a}_{3}$ can affect the fundamental voltage component in $\hat{D}_{x}$.

\section{E. Robustness Against Stator Resistance Error}

As a bonus advantage, the proposed PAAs based online inverter voltage drop compensation in Fig. 1 can also reject the disturbance in flux estimation caused by resistance error, and keep the sensorless system stable when an erroneous $\hat{R}$ is used.

When $t \in[33,100] \mathrm{s}$ in Fig. 11, the resistance value used in (8) is detuned to be $50 \%$ and $200 \%$ of the nominal value, at $t=33 \mathrm{~s}$ and $t=60 \mathrm{~s}$, respectively. At the instant when the resistance detuning happens, the $\tilde{\theta}_{d}$ waveform shows some spikes but converges to zero quickly. With the aid of the online adaptation of $\hat{a}_{2}$ and $\hat{a}_{3}$ and the associated voltage compensation $\hat{D}_{x}$, the sensorless system does not lose stability. Particularly, $\hat{a}_{2}$ converges to higher value when used resistance $\hat{R}$ is too small, and converges to lower value when used resistance $\hat{R}$ is too large.

\section{DISCUSSION}

This section provides discussion on experiment in terms of disturbance study, comparative study, and sensitivity study.

\section{A. Analysis of the Disturbances}

Disturbances are observed in the profiles of position error $\tilde{\theta}_{d}$, speeds $\omega_{r}, \hat{\omega}_{r}$ and $q$-axis current $i_{q}$ in Fig. 10 and Fig. 11. We are now going to investigate the transient disturbances via zoomed-in plots, and studies the steady state periodic disturbances with Fourier analysis.

1) Transient Disturbances: To show the profiles of transient disturbances, the zoomed-in plots of Fig. 10 and Fig. 11 are presented in Fig. 12 and Fig. 13.

In Fig. 12, when dc bus voltage step changes to $150 \mathrm{~V}$, a large voltage error $\tilde{D}_{x}$ results, which disturbs position error $\tilde{\theta}_{d}$. As $\hat{a}_{2}$ and $\hat{a}_{3}$ are updated, $\tilde{\theta}_{d}$ converges towards zero, and the compensation voltages $\hat{D}_{\alpha}$ and $\hat{D}_{\beta}$ decrease remarkably in magnitude. The actual speed $\omega_{r}$ is drastically disturbed when $\omega_{\text {ob }}$ is low in Fig. 12a, but stays close to speed command when $\omega_{\text {ob }}$ is high in Fig. 12b. The comparison shows that the robustness of sensorless speed control is dependent on $\omega_{\mathrm{ob}}$. 


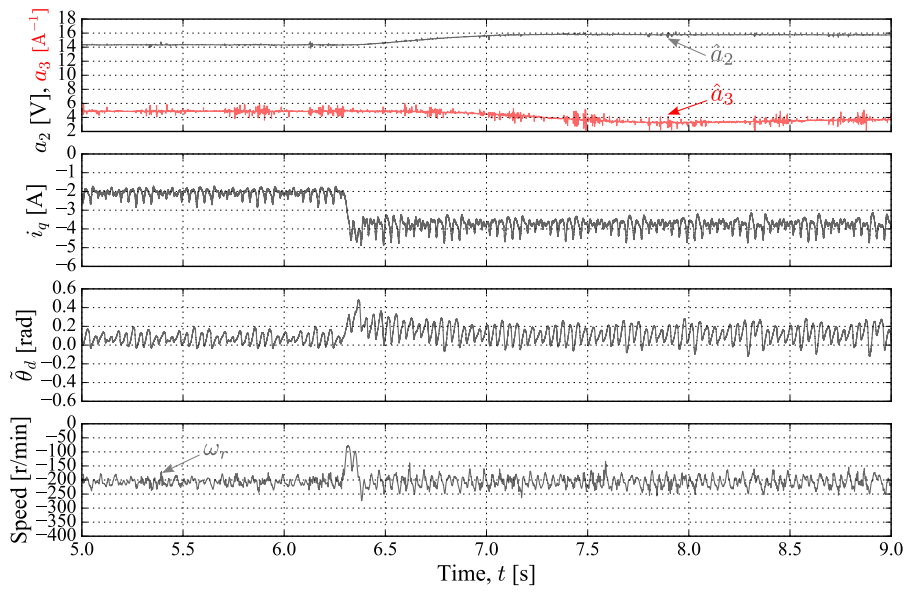

(a)

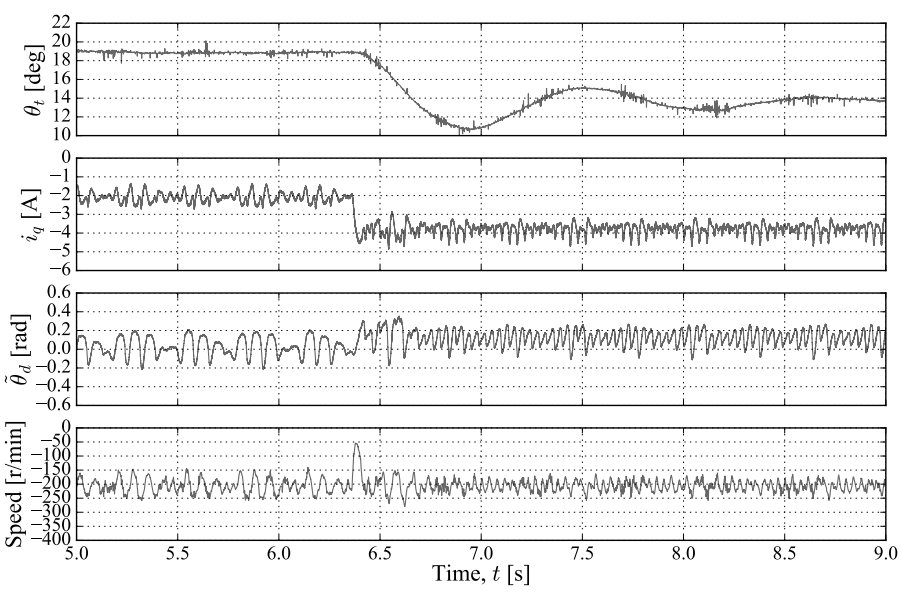

(b)

Fig. 15. Comparative experimental results when load changes: (a) proposed method, (b) existing method from [19].

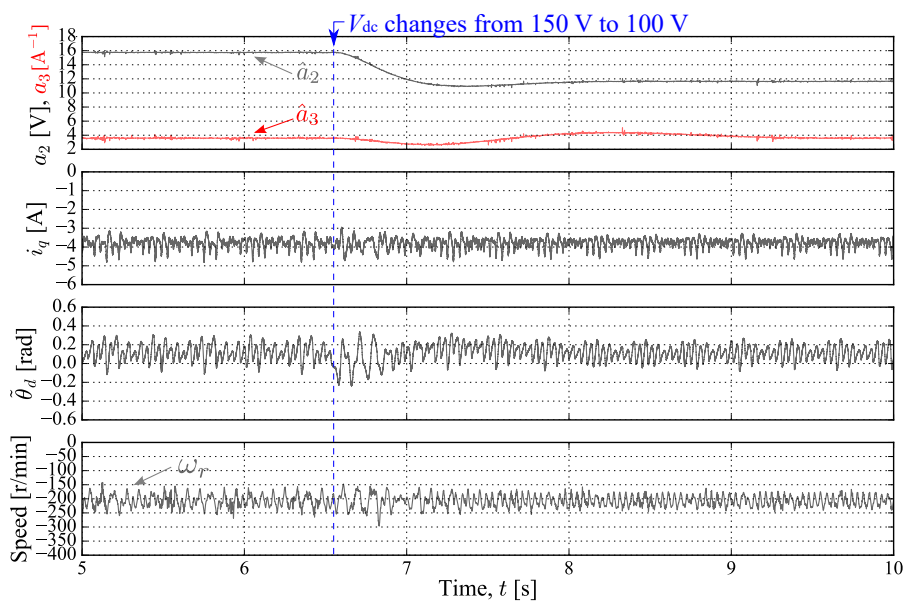

(a)

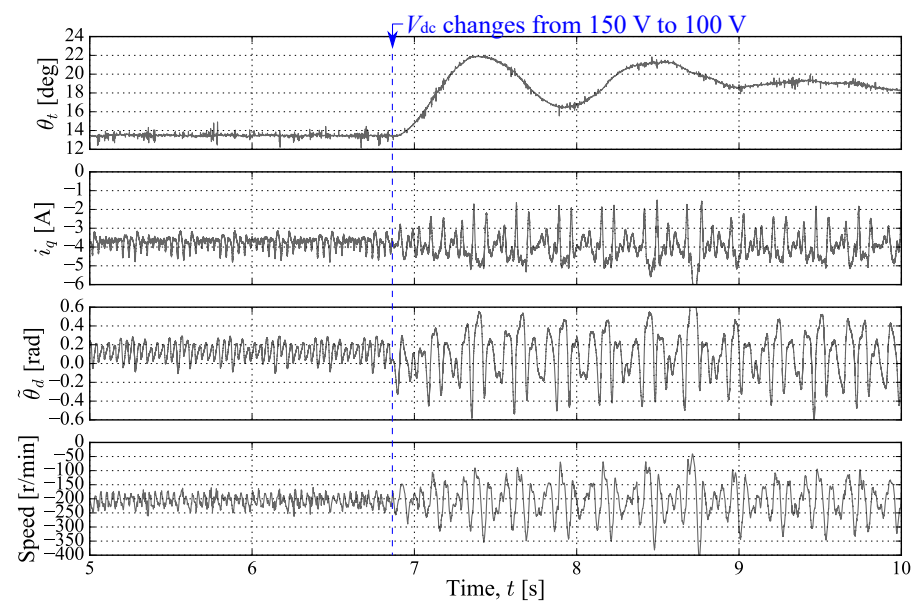

(b)

Fig. 16. Comparative experimental results when dc bus voltage $V_{\mathrm{dc}}$ changes: (a) proposed method, (b) existing method from [19].

In Fig. 13, when load changes, there is an undesired transient disturbance in $\tilde{\theta}_{d}, \omega_{r}$ and $\hat{\omega}_{r}$, which is caused by the voltage error corresponding to the transients of $\hat{a}_{2}$ and $\hat{a}_{3}$. Current amplitude $I_{x}$ is within LCR of the inverter, so $a_{2}$ and $a_{3}$ cannot be accurately identified because the parameter identifiability of $a_{2}$ and $a_{3}$ is coupled in LCR.

2) Steady State Periodic Disturbances: The Fourier analysis of steady state waveforms in Fig. 11 is shown in Fig. 14. From Fig. 14, the following observations can be made:

- The speed disturbance at synchronous frequency $\left(f_{1}=\right.$ $13.3 \mathrm{~Hz}$ ) is due to dc offset in measured current [25].

- The speed disturbance at $2 f_{1}, 4 f_{1}, 6 f_{1}$ is due to stator manufacturing tolerances [27].

- The speed disturbance at $4.5 f_{1}=60 \mathrm{~Hz}$ is due to rotor manufacturing tolerances, which implies the 4 pole-pair servo motor has 18 slots, resulting in the $\left(k \times \frac{18}{4}\right)$-th order harmonics in speed [27].

- The speed disturbance at $2.25 f_{1}=30 \mathrm{~Hz}$ is due to stator and rotor manufacturing tolerances, according to our finite element simulation studies of an 18-slot, 8-pole motor with both manufacturing tolerances at both stator teeth and rotor magnets.
- The speed disturbance at $11.6 \mathrm{~Hz}, 15 \mathrm{~Hz}, 23.2 \mathrm{~Hz}$ is due to sensorless control, because those harmonics are absent in $\omega_{r}$ when motor is controlled using encoder.

\section{B. Comparative Study}

This subsection conducts comparative study to further highlight the advantages of the proposed online adaptation method. To this end, we have implemented the trapezoidal compensation voltage method proposed in [19] for comparison. The experimental results are shown in Fig. 15 and Fig. 16. When implementing the method in [19], a fixed plateau voltage $\hat{a}_{2}$ is used, which is only accurate at $V_{\mathrm{dc}}=150 \mathrm{~V}$; and its shape parameter, i.e., the ramp region angle $\theta_{t} \in\left[0^{\circ}, 25^{\circ}\right]$ is defined in [19].

From Fig. 15, when $i_{q} \approx-2 \mathrm{~A}$ and $\theta_{t}=19^{\circ}$, it is observed that the peak-to-peak value of position error $\tilde{\theta}_{d}$ of the proposed method is approximately $0.2 \mathrm{rad}$, which is almost half of that of the existing method. This is because large $\theta_{t}$ is causing much loss in fundamental component of $\hat{D}_{x}$. A step load change is applied at $t \approx 6.3 \mathrm{~s}$, and both methods show similar performance as $i_{q}$ has become large enough for INV2, which can be understood by the fact that when current is high enough, 


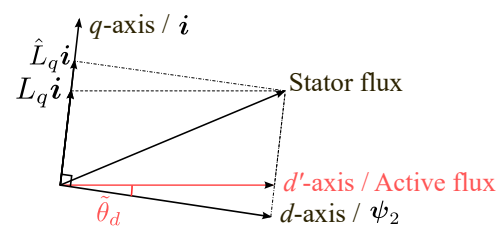

(a)

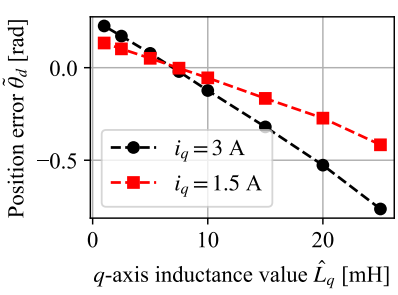

(b)
Fig. 17. (a) The phasor diagram that reveals positive position error $\tilde{\theta}_{d}$ due to $\hat{L}_{q}>L_{q}$ with $i_{d}=0$ control. (b) Measured position error $\tilde{\theta}_{d}$ versus the value $\hat{L}_{q}$ used in the active flux estimator (8).

the shape of the compensation voltage for both methods will approach a square waveform.

Fig. 15 shows that a change in the shape parameter $\left(\hat{a}_{3}\right.$ or $\theta_{t}$ ) often leads to a change in fundamental component of $\hat{D}_{x}$. For our proposed method, the fundamental voltage error is actively compensated by online adaptation of $\hat{a}_{2}$, but for method from [19], the fundamental voltage error is not controlled and becomes larger when $\theta_{t}$ is larger, causing larger peak-to-peak value of $\tilde{\theta}_{d}$ in Fig. $15 \mathrm{~b}$.

In Fig. 16a, for the proposed method, the peak-to-peak value of $\tilde{\theta}_{d}$ is less than $0.4 \mathrm{rad}$, and there is no apparent difference before and after $V_{\mathrm{dc}}$ changes, because the change in distortion voltage $D_{x}$ is compensated by online adaptation of $\hat{a}_{2}$. As a comparison, in Fig. 16b, $\theta_{t}$ is updated to mitigate voltage error due to erroneous plateau voltage when $V_{\mathrm{dc}}$ drops to $100 \mathrm{~V}$, but the sensorless system falls into oscillation giving a peakto-peak value of $\tilde{\theta}_{d}$ that is over $1.2 \mathrm{rad}$.

\section{Sensitivity Study}

This subsection discuss experimental behavior due to $L_{q^{-}}$ uncertainty, dead-time settings, and speed commands.

1) Parameter Uncertainty in $L_{q}$ : In Sec. VI-B, we have chosen to use the peak-to-peak value of position error $\tilde{\theta}_{d}$ as performance metric, rather than the mean value of $\tilde{\theta}_{d}$. This is because the mean value of $\tilde{\theta}_{d}$ is dependent on uncertainty in $L_{q}$. As is shown in Fig. 17a, when the $q$-axis inductance value $\hat{L}_{q}$ we used in the controller is different from the actual one $L_{q}$, position error $\tilde{\theta}_{d}$ will result.

The experimental measurement of $L_{q}$-uncertainty is shown in Fig. 17b, where the mean position error is plotted against the $L_{q}$-uncertainty under two different current levels. The influence of $L_{q}$-uncertainty on position estimation accuracy is found to be dependent on motor current.

Another issue due to $L_{q}$-uncertainty we find from Fig. 17a is that the projection of $\boldsymbol{i}$ to $d^{\prime}$-axis is not zero, denoted by $i_{d^{\prime}}$, meaning that there is a change in the actual active flux amplitude $K_{\text {Active }}=K_{E}+\left(L_{d}-L_{q}\right) i_{d^{\prime}}$ if $L_{d} \neq L_{q}$. This will affect our saturation function based flux estimator whose limit $\ell$ is calculated as $\ell=K_{E}+\left(L_{d}-L_{q}\right) \times 0$ A. From Fig. $17 \mathrm{~b}$, it is found that the $\hat{L}_{q}$ value that minimizes the mean position error is $7.5 \mathrm{mH}$ which is larger than $L_{d}$. This implies $\ell$ does not equal to $K_{\text {Active }}$ in our experiment. But the test motor has small inductance so the change in $K_{\text {Active }}$ is ignored in our study.

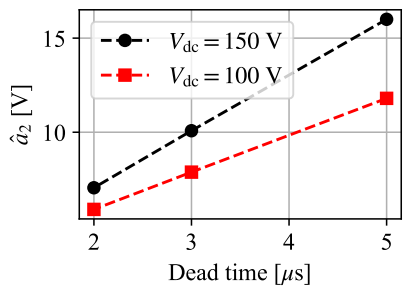

(a)

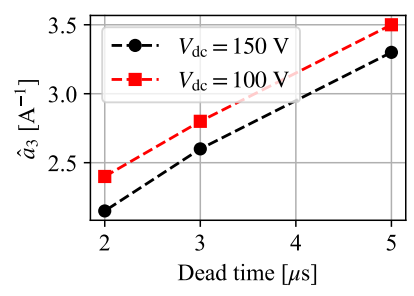

(b)
Fig. 18. Measured online identified inverter model parameters values versus dead-time, under two different dc bus voltages: (a) $\hat{a}_{2}$, (b) $\hat{a}_{3}$.

2) Different Dead-Time Settings: More experimental results are performed to record estimated $\hat{a}_{2}, \hat{a}_{3}$ values under different dead-time settings, as shown in Fig. 18. It is found that both $\hat{a}_{2}, \hat{a}_{3}$ increase as the dead-time increases. According to [20], one realize the slope of the curves in Fig. 18a is approximately proportional to $V_{\mathrm{dc}}$ times dead-time.

3) Lowest Operating Speed: According to our experiment, the sensorless drive is able to stably operate down to $100 \mathrm{r} / \mathrm{min}$ when $i_{q}$ is $3 \mathrm{~A}$, or $150 \mathrm{r} / \mathrm{min}$ when $i_{q}$ is $4 \mathrm{~A}$.

\section{CONCLUding Remarks}

This paper has been following the design process for a generic parameter adaptive system:

1) Model the phenomenon based on the physics, or at least provide an approximated way of representation.

2) Decide which parameters need to be online identified.

3) Study the single parameter-identifiability. The most intuitive way is to look at which variables show "abnormal behaviors" when the single parameter is erroneous. The PAAs are dynamics to minimize the error indicators.

4) If there is more than one unknown parameter, the coupled identifiability must be analyzed. Luckily, if there is only one-way coupling, prioritized adaptation rates should suffice; whereas unfortunately, if there is twoway coupling, it is suggested to not simultaneously identify these two parameters.

5) Close the loop by using the online updated parameters.

This procedure has been executed in this paper as follows:

1) The inverter voltage drop is modeled using sigmoid function for engineering purposes.

2) The plateau voltage $a_{2}$ due to dead-time and the shape parameter $a_{3}$ due to stray capacitor are to be identified.

3) The error indicators, i.e., $m B$ and $I_{\Sigma h}$ are built upon the abnormally behaved variables, i.e., $\psi_{2}$ and $i$. In particular, it is revealed that a nonlinear flux estimator must be used to build an effective error indicator $m B$. PAAs are designed to reduce $m B$ and $I_{\Sigma h}$.

4) When motor's peak current $I_{x}$ is within inverter's low current region, two-way coupling becomes severe; but if $I_{x}$ is large enough, it is valid to assume only one-way coupling exists between the identifiability of $a_{2}$ and $a_{3}$.

5) The identified $\hat{a}_{2}$ and $\hat{a}_{3}$ are used to compensate inverter voltage drop.

The proposed PAAs based inverter compensation scheme improves the robustness of sensorless algorithm against not 
only voltage error but also resistance error. It is theoretically suggested to suspend the PAAs when operating in inverter's low current region. However, as have been shown with experimental results, given the fact that the inverter current rating is $120 \mathrm{~A}$ and the motor current rating is only 3 Arms, the PAAs still work, even though the inverter parameters might never converge to their actual values anymore. This result raises a question to engineers designing the adaptive systemwhich is more desired, the accurate parameter identification or the robustness against uncertainty (of dc bus voltage and stator resistance)? This paper points out that both accuracy and robustness can be preserved by selecting a motor whose current rating matches inverter's current rating.

\section{REFERENCES}

[1] I. Boldea, M. C. Paicu, and G. Andreescu, "Active flux concept for motion-sensorless unified ac drives," IEEE Transactions on Power Electronics, vol. 23, no. 5, pp. 2612-2618, 2008.

[2] D. E. Salt, D. Drury, D. Holliday, A. Griffo, P. Sangha, and A. Dinu, "Compensation of inverter nonlinear distortion effects for signalinjection-based sensorless control," IEEE Transactions on Industry Applications, vol. 47, no. 5, pp. 2084-2092, 2011.

[3] R. Raute, C. Caruana, C. S. Staines, J. Cilia, M. Sumner, and G. M. Asher, "Analysis and compensation of inverter nonlinearity effect on a sensorless pmsm drive at very low and zero speed operation," IEEE Transactions on Industrial Electronics, vol. 57, no. 12, pp. 4065-4074, Dec 2010.

[4] L. M. Gong and Z. Q. Zhu, "A novel method for compensating inverter nonlinearity effects in carrier signal injection-based sensorless control from positive-sequence carrier current distortion," IEEE Transactions on Industry Applications, vol. 47, no. 3, pp. 1283-1292, May 2011.

[5] Q. Wang, N. Zhao, G. Wang, S. Zhao, Z. Chen, G. Zhang, and D. G. Xu, "An offline parameter self-learning method considering inverter nonlinearity with zero-axis voltage," IEEE Transactions on Power Electronics, pp. 1-1, 2021.

[6] W. Sun, J. Gao, X. Liu, Y. Yu, G. Wang, and D. Xu, "Inverter nonlinear error compensation using feedback gains and self-tuning estimated current error in adaptive full-order observer," IEEE Transactions on Industry Applications, vol. 52, no. 1, pp. 472-482, 2015.

[7] S. Bolognani, L. Peretti, and M. Zigliotto, "Repetitive-control-based selfcommissioning procedure for inverter nonidealities compensation," IEEE Transactions on Industry Applications, vol. 44, no. 5, pp. 1587-1596, Sept 2008.

[8] S. Bolognani and M. Zigliotto, "Self-commissioning compensation of inverter non-idealities for sensorless ac drives applications," in Power Electronics, Machines and Drives, 2002. International Conference on (Conf. Publ. No. 487), June 2002, pp. 30-37.

[9] J.-H. Lee and S.-K. Sul, "Compensation of nonlinearity of inverter through estimation of dead time effect," in 2020 IEEE 9th International Power Electronics and Motion Control Conference (IPEMC2020-ECCE Asia). IEEE, 2020, pp. 572-577.

[10] H.-S. Kim, K.-H. Kim, and M.-J. Youn, "On-line dead-time compensation method based on time delay control," IEEE Transactions on Control Systems Technology, vol. 11, no. 2, pp. 279-285, 2003.

[11] H.-S. Kim, H.-T. Moon, and M.-J. Youn, "On-line dead-time compensation method using disturbance observer," IEEE Transactions on Power Electronics, vol. 18, no. 6, pp. 1336-1345, 2003.

[12] Y. Wang, Y. Xu, and J. Zou, "Ilc-based voltage compensation method for pmsm sensorless control considering inverter nonlinearity and sampling current dc bias," IEEE Transactions on Industrial Electronics, vol. 67, no. 7 , pp. 5980-5989, July 2020.

[13] Z. Tang and B. Akin, "A new Ims algorithm based deadtime compensation method for pmsm foc drives," IEEE Transactions on industry applications, vol. 54, no. 6, pp. 6472-6484, 2018.

[14] D. Liang, J. Li, R. Qu, and W. Kong, "Adaptive second-order slidingmode observer for pmsm sensorless control considering vsi nonlinearity," IEEE Transactions on Power Electronics, vol. 33, no. 10, pp. 89949004, Oct 2018.

[15] Y. Inoue, K. Yamada, S. Morimoto, and M. Sanada, "Effectiveness of voltage error compensation and parameter identification for modelbased sensorless control of ipmsm," IEEE Transactions on Industry Applications, vol. 45, no. 1, pp. 213-221, 2009.
[16] Y. Park and S. K. Sul, "A novel method utilizing trapezoidal voltage to compensate for inverter nonlinearity," IEEE Transactions on Power Electronics, vol. 27, no. 12, pp. 4837-4846, Dec 2012.

[17] S.-H. Hwang and J.-M. Kim, "Dead time compensation method for voltage-fed pwm inverter," IEEE Transactions on Energy Conversion, vol. 25 , no. 1 , pp. $1-10,2010$.

[18] K. Liu and Z.-Q. Zhu, "Online estimation of the rotor flux linkage and voltage-source inverter nonlinearity in permanent magnet synchronous machine drives," IEEE Transactions on Power Electronics, vol. 29, no. 1, pp. 418-427, 2013.

[19] Y. Park and S. K. Sul, "Implementation schemes to compensate for inverter nonlinearity based on trapezoidal voltage," IEEE Transactions on Industry Applications, vol. 50, no. 2, pp. 1066-1073, March 2014.

[20] J.-W. Choi and S.-K. Sul, "Inverter output voltage synthesis using novel dead time compensation," IEEE Transactions on Power Electronics, vol. 11, no. 2, pp. 221-227, Mar 1996.

[21] S.-K. Sul, Control of electric machine drive systems. John Wiley \& Sons, 2011, vol. 88.

[22] J.-S. Kim, J.-W. Choi, and S.-K. Sul, "Analysis and compensation of voltage distortion by zero current clamping in voltage-fed pwm inverter," IEEJ Transactions on Industry Applications, vol. 117, no. 2, pp. 160165,1997

[23] A. Munoz and T. Lipo, "On-line dead-time compensation technique for open-loop pwm-vsi drives," IEEE Transactions on Power Electronics, vol. 14 , no. 4 , pp. 683-689, 1999.

[24] J. Chen, J. Mei, X. Yuan, Y. Zuo, and C. H. T. Lee, "Natural speed observer for nonsalient ac motors," IEEE Transactions on Power Electronics, pp. 1-1, 2021.

[25] J. Holtz and J. Quan, "Drift- and parameter-compensated flux estimator for persistent zero-stator-frequency operation of sensorless-controlled induction motors," IEEE Transactions on Industry Applications, vol. 39, no. 4, pp. 1052-1060, July 2003.

[26] Y. Zuo, J. Chen, X. Zhu, and C. H. T. Lee, "Different active disturbance rejection controllers based on the same order gpi observer," IEEE Transactions on Industrial Electronics, 2021.

[27] J. Ou, Y. Liu, R. Qu, and M. Doppelbauer, "Experimental and theoretical research on cogging torque of pm synchronous motors considering manufacturing tolerances," IEEE Transactions on Industrial Electronics, vol. 65, no. 5, pp. 3772-3783, 2018.

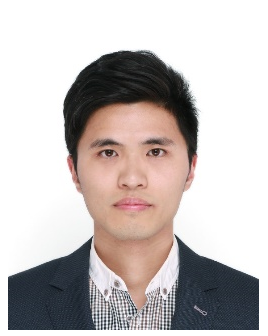

Jiahao Chen (S'17-M'19) received the B.Sc. and $\mathrm{Ph} . \mathrm{D}$. degrees in electrical engineering from $\mathrm{Zhe}$ jiang University, China, in 2014 and 2019, respectively. Since September 2018, he was a visiting scholar at the University of Wisconsin-Madison, USA for one year, where he was involved in bearingless motors. He is currently a post doctoral research fellow with Nanyang Technological University, Singapore. His research interests include electric machines, drives, and direct-drive technologies.

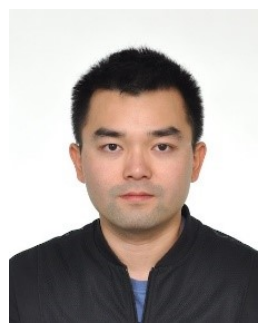

Jie Mei (M'13) received his B.S. degree in electrical engineering from the School of Electrical and Computer Engineering, Georgia Institute of Technology, Atlanta, GA, USA, in 2015, and the Ph.D. degree in electrical engineering from the Department of Electrical Engineering and Computer Science, Massachusetts Institute of Technology, Cambridge, MA USA, in 2021 . He is currently a research specialist with the Department of Electrical Engineering and Computer Science, Massachusetts Institute of Technology, Cambridge, MA, USA. His research interests include Electric Machines, Multi-Energy Systems, and Electric Vehicle Technologies. 


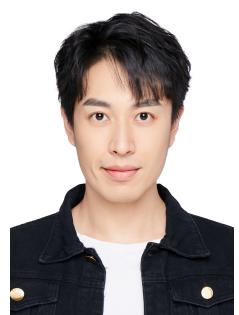

Xin Yuan (S'19-M'20) was born in Heilongjiang, China, in 1990. He received the B.S. and M.S degrees in 2013 and 2016, respectively. In 2020, he received the $\mathrm{PhD}$ degrees in electrical engineering from Beijing Institute of Technology, Beijing, China. He is currently a Research fellow with the School of Electrical and Electronic Engineering, Nanyang Technological University, Singapore. He was a research associate in PEMC group, University of Nottingham, UK from Jan. 2019 to Jan. 2020. His research interests include $\mathrm{AC}$ motor drives, power converters, multi-phase motor drives and faulttolerant strategy of motor.

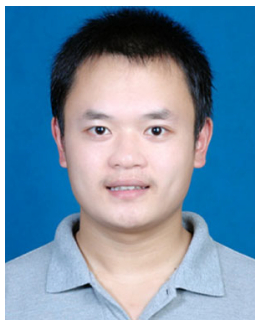

Yuefei Zuo (M'18) received the B.Sc. and the Ph D. degrees in electrical engineering and automation from Nanjing University of Aeronautics and Astronautics, Nanjing, China, in 2010 and 2016, respectively. He has been working as a Lecturer at Jiangsu University since July 2016. He is currently a Postdoctoral Research Fellow with the School of Electrical and Electronic Engineering, Nanyang Technological University, Singapore. His research interests include power electronics, electric machines and drives, and advanced control strategies.

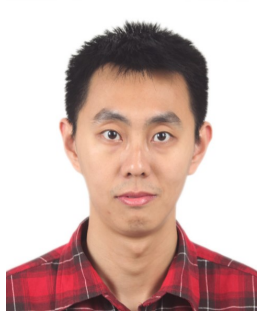

Jingwei Zhu received the B.Sc. degree in electrical engineering and automation from Zhejiang University, Hangzhou, China, in 2015 and the M.Phil. degree in electrical engineering from The Hong Kong Polytechnic University, Hong Kong, in 2018.

He was the Edward R. Felber Power Fellowship Scholar at the University of Wisconsin-Madison from 2018 to 2019 where he worked within the Wisconsin Electric Machines and Power Electronics Consortium (WEMPEC). From September 2019 to July 2020, he was a Research Associate within Center for System Intelligence and Efficiency (CSIE), Nanyang Technological University. From August 2020, he is currently working toward the Ph.D. degree in electrical and electronic engineering, Nanyang Technological University, Singapore. His research interests include motor design \& optimization, power electronics, bearingless motors and direct-drive techiques.

Mr. Zhu received the 2018 Edward R. Felber Power Fellowship awarded by University of Wisconsin-Madison.

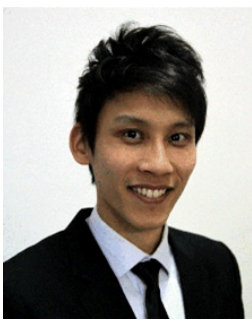

Christopher H. T. Lee (M'12-SM'18) received his B.Eng. (First Class Honours) degree, and Ph.D. degree both in electrical engineering from Department of Electrical and Electronic Engineering, The University of Hong Kong, Hong Kong.

He currently serves as an Assistant Professor at Nanyang Technological University, Singapore and Honorary Assistant Professor at The University of Hong Kong, Hong Kong. He was a Postdoctoral Fellow and then a Visiting Assistant Professor at Massachusetts Institute of Technology, United States. $\mathrm{He}$ is an Associate Editor for IEEE Transactions on Industrial Electronics, IEEE Transactions on Energy Conversion, IEEE Access and IET Renewable Power Generation. He is a Chartered Engineer in Hong Kong. His research interests include Electric Machines and Drives, Renewable Energies, and Electromechanical Propulsion Technologies. In these areas, he has published 1 book, 3 books chapters, and over 100 referred papers.

Dr. Lee has received many awards, including NRF Fellowship, Nanyang Assistant Professorship, Li Ka Shing Prize (the best Ph.D. thesis prize) and Croucher Foundation Fellowship. 